\title{
ON THE CLASSIFICATION OF SIMPLE ANTIFLEXIBLE ALGEBRAS
}

BY

\author{
MAHESH CHANDRA BHANDARI $\left(^{1}\right)$
}

ABSTRACT. In this paper, we begin a classification of simple totally antiflexible algebras (finite dimensional) over splitting fields of characteristic $\neq 2,3$. For such an algebra $A$ let $P$ be the largest associative ideal in $A^{+}$and let $N$ be the radical of $P$. We say that $A$ is of type $(m, n)$ if $N$ is nilpotent of class $m$ with $\operatorname{dim} A=n$. Define $N_{i}=N_{i-1} \cdot N, N_{1}=N$, then $A$ is said to be of type $\left(m, n, d_{1}, d_{2}, \cdots, d_{q}\right)$ if $A$ is of type $(m, n), \operatorname{dim}\left(N_{i}-N_{i-1}\right)=d_{i}$ for $1 \leq i \leq q$ and $\operatorname{dim}\left(N_{i}-N_{i+1}\right)=1$ for $q<i<m$. We then determine all nodal simple totally ant if lexible algebras of types $(n, n),(n-k, n, k+1),(n-2, n)$ (over fields of characteristic $\neq 2,3)$ and of type $(3,6)$ (over the field of complex numbers). We also give preliminary results for nodal simple totally antiflexible algebras of type $(n-k, n, k, 2)$ and of type $\left(m, n, d_{1}, \cdots, d_{q}\right)$ in general with $m>2$ (the case $m=2$ has been classified by D. J. Rodabaugh).

1. Introduction. A totally antiflexible algebra is a nonassociative algebra (finite dimensional) satisfying

$$
(x, y, z)=(z, y, x)
$$

and

$$
(x, x, x)=0
$$

where $(x, y, z)=(x y) z-x(y z)$. Totally antiflexible algebras have been studied by C. Anderson and D. Outcalt [1], F. Kosier [3] and D. Rodabaugh [4], [5], [6], [7], [8]. These are known to be related to the algebras of commutative nilpotent matrices [8]. There is not much known about the algebras of commutative nilpotent matrices. In this paper we complete the classification of simple nodal totally antiflexible algebras that are related to the algebras of commutative nilpotent matrices discussed by D. A. Suprunenko and R. I. Tyškevič [10] and certain other types.

Define $x^{1}=x, x^{k+1}=x^{k} x$ and $x^{\cdot 1}=x, x^{\cdot k+1}=x^{\cdot k} \cdot x$. It is known [6] that a totally antiflexible algebra need not be power-associative when char. $\not 0$. However, $A^{+}$is power-associative so $x^{\cdot m} \cdot x^{\cdot n}=x^{\cdot m+n}$ for all positive integers $m$ and $n$. We will call $y$ nilpotent or nil if $y^{\cdot n}=0$ for some $n$. If $x$ in $A$ implies

Presented to the Society, August 3, 1971; received by the editors August 19, 1971. AMS 1969 subject classifications. Primary 1760. algebras.

Key words and phrases. Simple algebras, antiflexible algebras, totally antiflexible

(1) This paper contains major results from the author's doctoral dissertation written under the supervision of Dr. D. J. Rodabaugh. 
$x=\alpha \cdot 1+z$ for $\alpha$ in the base field and $z$ nil, we say that $A$ is nearly nodal. A nearly nodal algebra is nodal if the set of nil elements do not form a subalgebra.

2. Preliminaries. We will state some known results on the structure of simple totally antiflexible algebras. We also need (see [1], [7])

Definition 2.1. A field $K$ is said to be a splitting field for an algebra $A$ if every primitive idempotent of $A_{K}$ is absolutely primitive and if every element in $\left(A_{K}\right)_{11}(e)$ for $e$ primitive can be written as $k e+y$ with $k$ in $K$ and $y$ nil or $y=0$.

Definition 2.2. Let $A$ be an algebra over a field $F$ of char. $\neq 2,3$. The mapping $\phi: A \times A \rightarrow B$ for $B \subseteq A$ will be called an antiflexible map provided $B \subseteq\{x: x y=y x$ for all $y$ in $A\}$ and

(3) $\phi$ is bilinear over $F$,

(4) $\phi(x, y)+\phi(y, x)=0$,

(5) $\phi\left(x^{2}, x\right)=0$,

(6) $\phi(x, y)=0$ if $y$ is in $B$,

(7) $\phi((x, y), z)=0$.

For $\alpha, \beta$ in $F$ and antiflexible maps $\phi_{1}, \phi_{2}$ define $a \phi_{1}+\beta \phi_{2}$ by

$$
\alpha \phi_{1}+\beta \phi_{2}(x, y)=\alpha \cdot \phi_{1}(x, y)+\beta \phi_{2}(x, y) \text {. }
$$

It is clear that $\alpha \phi_{1}+\beta \phi_{2}$ is an antiflexible map.

Definition 2.3. Let $A$ be an algebra over a field of char. $\neq 2,3$ and $\phi$ be an antiflexible map. Define $A(\phi)$ as the algebra formed from $A$ with multiplication replaced by

$$
x * y=x y+\phi(x, y) \text {. }
$$

It is known [4] that $A$ is totally antiflexible if and only if $A(\phi)$ is totally antiflexible. Furthermore, if $\psi$ is an antiflexible map on $A(\phi)$, then $A(\phi)(\psi)=$ $A(\phi+\psi)$.

We now summarize certain results in [1], [4] by the following theorem.

Theorem 2.1. If $A$ is a simple not associative totally antiflexible algebra, over a splitting field $F$ of char. $\neq 2,3$ then $A^{+}$is associative, $A$ bas an identity element and $A=A_{1}+\cdots+A_{n}$ where $A_{i}=A_{11}\left(e_{i}\right)$ for $e_{i}$ primitive. Further more, $\phi(x, y)=(1 / 2)(x, y)$ is an antiflexible map and $A=A^{+}(\phi)$.

We will then be interested in those algebras from which simple algebras can be constructed. We say that a totally antiflexible algebra $A$ is nearly simple if there is an antiflexible map $\phi$ such that $A(\phi)$ is simple.

Theorem 2.2 [8]. Let $A$ be a totally antiflexible algebra over a field of char. $\neq 2,3$ and assume $A^{+}$is associative. Then $A$ is nearly simple if and only if $A^{+}$is nearly simple. 
As a result of Theorems 2.1 and 2.2, to find all simple algebras, we need only consider the nearly simple associative and commutative algebras. These are known to contain an identity element. For, if $A$ is a nearly simple associative commutative algebra then there is an antiflexible map $\phi$ with $A(\phi)$ simple. So by Theorem 1.1, $A(\phi)$ contains an identity element. Therefore $A$ contains an identity element. Hence, throughout this paper unless specified we will assume that $A$ is a totally antiflexible algebra with identity element over a splitting field $K$ of char. $\neq 2,3$ and that $A^{+}$is associative. Consequently, $A=A_{1}+\cdots+A_{n}$ with $A_{i}=A_{11}\left(e_{i}\right)$ for $e_{i}$ primitive and $A_{i} A_{j}=0$ if $i \neq j$. For, since $A^{+}$is associative, $A_{10}(e)+A_{01}(e)=0$ for any idempotent $e$ (see also [5], [7]). In addition, since $K$ is a splitting field, each element in $A_{i}$ has the form $\alpha e_{i}+z$ for $a$ in $K$ and $z$ nil. Thus $A$ has a basis consisting of primitive idempotents and nil elements. We define the following sets:

(8) $N=\{x: x$ in $A$ and $x$ is nil $\}$,

(9) $N_{i}=N_{i-1} \cdot N$ with $N_{1}=N$,

(10) $\frac{i}{N_{i}}=N_{i}-N_{i+1}$ (quotient or difference algebra),

(11) $N_{i}^{\prime}=\left\{x: x\right.$ is in $N_{i}$ but not in $\left.N_{i+1}\right\}$,

(12) $M_{i}=\left\{x: x \cdot N \subseteq M_{i-1}\right\}$ with $M_{0}=0$. $M_{1}(=M)$ is called the annihilator of $N$.

For each $x$ in $A^{+}$define $T_{x}: y \rightarrow y \cdot x$ and note that, since there is an identity element 1 in $A$ and $A^{+{ }^{x}}$ is associative, $x \rightarrow T_{x}$ is an isomorphism of $A^{+}$onto $\left\{T_{x}\right\}$. Thus, if $\operatorname{dim} A=n$, we can think of either $A$ or one of its subalgebras as an algebra of commutative $n \times n$ matrices.

For some $m, N_{m}=0$ with $N_{m-1} \neq 0$ and so $N_{1} \supseteq N_{2} \supseteq \cdots \supseteq N_{m}=0=M_{0} \subseteq$ $M_{1} \subseteq \cdots \subseteq M_{m-1} \subseteq M_{m}=N$ and $N_{i} \subseteq M_{m-i}$ for all $i$. We say that $A$ (or $N$ ) is of type $(m, n)$ if $A^{+}$(or $N^{+}$) is isomorphic to an algebra of commutative $n \times n$ matrices for $n=\operatorname{dim} A$ with $N_{m}=0 \neq N_{m-1}$. The algebra $A$ (or $N$ ) is said to be of class $m$. An algebra $A$ (or $N$, the radical of $A^{+}$) is of type $\left(m, n, d_{1}, \ldots, d_{q}\right.$ ) if $A$ (or $N$ ) is of type $(m, n), \operatorname{dim} \bar{N}_{i}=d_{i}$ for $1 \leq i \leq q$ and $\operatorname{dim} \bar{N}_{i}=1$ for $q<$ $i \leq m-1$. Note that if $N_{i}=N_{i+1}$ then $N_{i}=N_{j}$ for all $j \geq i$. Hence either $N_{i}=0$ or $\operatorname{dim} \overline{N_{i}} \geq 1$. It is known [8] that if $\operatorname{dim} \bar{N}_{i}=1$ for some $i$ then $\operatorname{dim} \overline{N_{i+k}}=1$ for $k=0, \cdots, m-i-1$. Hence we can assume that $d_{i}>1$ for $1 \leq i \leq q$.

By Theorems 2.1 and 2.2 the problem of classifying all simple totally antiflexible algebras is reduced to finding

(i) a characterization of all nearly simple associative commutative algebras,

(ii) all possible antiflexible maps $\phi$ that give rise to simple antiflexible algebras.

The following two theorems summarize results from [8].

Theorem 2.3. Let $P$ be an associative, commutative algebra over a field of char. $\neq 2,3$ and let $\phi$ be a bilinear map from $P \times P \rightarrow B \subseteq P$ such that $\phi(P, B)=0$. 
Then $\phi$ is an antiflexible map if and only if, for every $n ; y_{1}, \ldots, y_{n}$,

$$
\sum_{j=1}^{n} \phi\left(\pi_{i \neq j} y_{i}, y_{j}\right)=0 .
$$

If $x$ is in $M_{i}, y$ in $N_{j}, z$ in $N_{j+1}, j \geq i \geq 1$, and $\phi$ an antiflexible map, then $x \cdot y=0$ and $\phi(x, z)=0[8]$. Now we state necessary and sufficient conditions for a totally antiflexible algebra to be simple.

Theorem 2.4. Let $A$ be a totally antiflexible algebra over a splitting field of char. $\neq 2,3$ with $A^{+}$associative. Then $A$ is simple if and only if

(14) for every nonzero $x$ in $M_{1}$ there is a $y$ in $N$ with $(x, y) \neq 0$,

(15) no element of $\{e(x, y)\}$ generates a proper ideal where $e$ is a primitive idempotent,

(16) for each primitive idempotent $e$ in $A,\{e(x, y)\}$ is not nil.

The proof of the following theorem is similar to that of Theorem 2.4.

Theorem 2.5. Let $A$ be a totally antiflexible nodal algebra, over a field of char. $\neq 2,3$ with $A^{+}$associative. Then $A$ is simple if and only if

(17) for every nonzero $x$ in $M_{1}$ there is a $y$ in $N$ with $(x, y) \neq 0$,

(18) for each $x$ in $M_{1}$ and $y$ in $N_{1}^{\prime},(x, y)$ does not generate a proper ideal of $A$.

Proof. If $A$ is simple, then the conclusion follows from Theorem 2.4.

Conversely, suppose $A$ satisfies (17) and (18) and $J$ is a proper ideal of $A$. Let $x$ be in $J, x \neq 0$; then $x=a \cdot 1+z$ for some $\alpha$ in $F$ and $z$ nil. If $a \neq 0$, write $u=(-1 / \alpha)$. Then for some $m_{b} u^{\cdot m}=0 \neq u^{\cdot(m-1)}$ and so $1=(1-u)$ $\cdot\left(1+u+\cdots+u^{\cdot(m-1)}\right)$ is in $J$. Hence $J=A$ which is impossible. Therefore suppose $\alpha=0$. If $z$ is in $M$, let $u=z$ and if $z$ is not in $M_{1}$, then there is a $y$ in $N$ with $u=z \cdot y$ in $M_{1} \cap J$. Hence by (17) there is a $v$ in $N$ such that $(u, v)$ $\neq 0$ and $(u, v)$ is in $J$. Therefore by (18) it follows that $J=A$, which is a contradiction. Consequently $A$ is simple.

In a similar way we can prove the following.

Lemma 2.1. If $A$ is a nodal totally antiflexible algebra over a field of char. $\neq 2,3$ and if for $\phi(x, y)=(1 / 2)(x, y), N \cap\{\phi(x, y)\}=0$, then $A$ is simple if and only if for every nonzero $x$ in $M_{1}$ there exists $a y$ in $N$ sucb that $\phi(x, y) \neq 0$.

Proof. We only need to prove that the condition is sufficient. Hence suppose that for every nonzero $x$ in $A$ there is a $y$ in $N$ such that $\phi(x, y) \neq 0$ and $J$ is a proper ideal of $A$. Let $x$ be in $A, x \neq 0$. Then $x=a \cdot 1+z$ with $\alpha$ in $F$ and $z$ nil. If $\alpha \neq 0$, define $u=(-1 / \alpha) z$, then for some $m,(1 / \alpha) x \cdot\left(1+u+\cdots+u^{\cdot m}\right)=1$ 
is in $J$ which is impossible. Therefore, suppose that $\alpha=0$, for every $x$ in $J$ and $J \subseteq N$. Let $x$ be in $J, x \neq 0$. If $x$ is not in $M$, then for some $x, x \cdot z$ is in $M$, and so there is a $y$ with $\phi(x \cdot z, y) \neq 0$. But then $\phi(x \cdot z, y)$ is not nil with $\phi(x \cdot z, y)$ in $J$, which contradicts the fact that $J \subseteq N$. Hence $A$ is simple.

For an antiflexible map $\phi$ on an algebra $A$ we define

$$
H(\phi)=\{\phi(x, y): x, y \text { are in } A\} \text { and }(x \phi y)=x * y-y * x
$$

where $x * y=x y+\phi(x, y)$. Thus $(x \phi y)=(x, y)+2 \phi(x, y)$.

If $H(\phi)=Z(A)$ (the center of $A$ ) and if $Z(A) \neq\{0\}$ then $Z(A)$ is a field [9]. Hence $H(\phi) \cap N=Z(A) \cap N=\{0\}$ and we have proved the following lemma.

Lemma 2.2. Let $A$ be a nodal totally antiflexible algebra over a splitting field $F$ of char. $\neq 2,3$. If $H(\phi)=Z(A)$ then $H(\phi) \cap N=\{0\}$.

The following two theorems summarize the results on algebras of class 2 [8].

Theorem 2.6. Let $P$ be a nearly nodal associative commutative algebra over a field of char. $\neq 2,3$ with $N \cdot N=0$. Let $\left\{x_{i}\right\}_{i=1}^{n}$ be a basis for $N$. If $\phi$ is an antiflexible map them $P(\phi)$ is simple if and only if there is a nonsingular matrix $X=\left(x_{i, j}\right)$ with $\phi\left(x_{i}, x_{j}\right)=x_{i, j}$

Theorem 2.7. Let $P$ be an associative commutative algebra over a splitting field $F$ with $N \cdot N=0$. Then $P$ is nearly simple if and only if

(19) there is an identity element in $P$,

(20) for every primitive idempotent $e, \operatorname{dim} P_{11}(e) \geq 3$,

(21) either 1 is not primitive or $\operatorname{dim} P$ is odd.

Next, three theorems summarize results on algebras of types $(n, n)$ and $(n-k, n, k+1)$ from [8].

Theorem 2.8. Let $N$ be an associative commutative nilalgebra of dimension $n-1$ over a field $F$. If $N$ is of class $m$ and if char $F=0$ or char $F \geq m$ or char $F \geq n-m+2$ or $\operatorname{dim} \overrightarrow{N_{k}}=1$ with char $F>k$ then there is an $x$ in $N$ with $x^{m-1} \neq 0$.

Theorem 2.9. Let $P=F \cdot 1 \oplus N$ where $N$ is an associative commutative nilalgebra of type $(n, n)$ over a field $F$ of char. $\neq 2,3$. Then $P$ is nearly simple if and only if char $F$ divides $n$.

Theorem 2.10. Let $P=F \cdot 1 \oplus N$ where $N$ is an associative commutative nilalgebra of type $(n-k, n, k+1)$ with $n-k>2$ over a field $F$ of char. $\neq 2,3$. The algebra $P$ is nearly simple if and only if the following bolds:

(22) $N$ is spanned by $a, \cdots, a^{n-k-1}, b_{1}, \cdots, b_{k}$ where $a b_{i}=b_{i} b_{j}=0 ; i$, $j=1,2, \cdots, k$, 
(23) either $n-k=$ char $F$ with $k$ even or $n-k=m$ char $F$ for $m>1$.

3. Nodal algebras of type $(n, n),(n-k, n, k+1)$ and $(n-2, n)$. We now focus attention on nodal algebras. If $A$ is such an algebra then $\operatorname{dim} A=1+\operatorname{dim} N$. Since all simple antiflexible algebras of class 2 have been determined [8], in classifying simple antiflexible algebras of type $(m, n)$, we can assume $m>2$. The following theorem gives an answer to the second question for algebras of type $(n, n)$. Notationally we use $a^{0}$ for 1 .

Theorem 3.1. Let $P=F \cdot 1 \oplus N$ be a nearly simple, associative, commuta tive, nearly nodal algebra of type $(n, n)$ over a field of $F$ of char. $\neq 2,3$. If $\phi$ is an antiflexible map on $P$ and if $a$ is chosen in $N$ so that $a^{n-1} \neq 0$, then $P(\phi)$ is simple if and only if

(24) $H(\phi)$ is a subset of the algebra generated by 1 and $a^{p}$, and $n=m p$,

(25) $\phi\left(a^{n-1}, a\right)=\Sigma_{i=0}^{m-1} \beta_{i p} a^{i p}, \beta_{i p}$ in $F$ with $\beta_{0} \neq 0$.

Proof. By Theorems 2.8 and 2.9 (see also Theorem 5.3 of [8]) there is an element $a$ in $P$ such that $P$ is generated by 1 and $a$ and $n=m p$ for some positive integer $m$. Also using Theorem 2.3 we have $\phi\left(a^{i}, a^{j}\right)=j \phi\left(a^{i+j+1}, a\right)=$ - $i \phi\left(a^{i+j-1}, a\right)$. Hence, $\phi\left(a^{i}, a^{j}\right)=0$ if either $p \mid i(p$ divides $i)$ or $p \mid j$ or $p \nmid(i+j)$ or $i+j>n$.

Now suppose $P(\phi)$ is simple. Then $\phi\left(a^{n-1}, a\right) \neq 0$. Let $x, y$ be in $P$. Then

$$
\phi(x, y)=\sum_{i=0}^{n-1} a_{i} a^{i}, \quad \alpha_{i} \text { in } F, i=0, \cdots, n-1 .
$$

Since $\phi$ is an antiflexible map on $P$, we have $0=\phi\left(\phi(x, y), a^{n-1}\right)=\alpha_{1} \phi\left(a, a^{n-1}\right)$. But $\phi\left(a^{n-1}, a\right) \neq 0$, so $\alpha_{1}=0$. Suppose $\alpha_{i}=0$ for all $i<k$ with $p \nmid i$ and $p \nmid k$. Then

$$
0=\phi\left(\phi(x, y), a^{n-k}\right)=\alpha_{k} \phi\left(a^{k}, a^{n-k}\right)=-k \alpha_{k} \phi\left(a^{n-1}, a\right),
$$

which implies that $\alpha_{k}=0$. Hence by mathematical induction, $\alpha_{i}=0$ for all $i$ with $\not \nmid i$ and so $\phi(x, y)=\sum_{i=0}^{m-1} \alpha_{i p} a^{i p}$. This proves (24).

If $x=a^{n-1}$ and $y=a$, we get $\phi\left(a^{n-1}, a\right)=\sum_{i=0}^{m-1} \beta_{i p} a^{i p}, \beta_{i p}$ in $F$. If $\beta_{0}$ $=0$, then $a$ generates a proper ideal of $P(\phi)$. Hence $\beta_{0} \neq 0$ and so (25) is satisfied.

Conversely, suppose $\phi$ satisfies (24) and (25) and $J$ is an ideal of $P(\phi)$. Let $x$ be in $J, x \neq 0$, then $x=\sum_{i=1}^{n-1} \alpha_{i} a^{i}, \alpha_{i}$ in $F, i=1, \cdots, n-1$. Let $j$ be the least integer such that $a_{j} \neq 0$. If $p ! j$ then

$$
\phi\left(x * a, a^{n-j-1}\right)=\phi\left(x a, a^{n-j-1}\right)=-(j+1) \alpha_{j}\left(\sum_{i=0}^{m-1} \beta_{i p} a^{i p}\right) .
$$

Therefore $\left(-1 /(j+1) \alpha_{j} \beta_{0}\right) \phi\left(x a, a^{n-j-1}\right)=1-z$ is in $J, z$ is nil. 
On the other hand, if $p \nmid j$ then

$$
\left(-1 / j a_{j} \beta_{0}\right) \phi\left(x, a^{n-j}\right)=\left(-1 / j \beta_{0}\right) \phi\left(a^{j}, a^{n-j}\right)=1-z
$$

is in $J$ for some $z ; z$ is nil. Hence in either case for some positive integer $r$, $(1-z)\left(1+z+\cdots+z^{r}\right)=1$ is in $J$. Therafore $J=P(\phi)$ and so $P(\phi)$ is simple.

In a similar way we prove the following theorem.

Theorem 3.2. Let $P=F \cdot 1 \oplus N$ be a nearly simple, associative commutative nearly nodal algebra of type $(n-k, n, k+1), n-k>2$, over a field $F$ of characteristic $p \neq 2,3$. Let $\phi$ be an antiflexible map and let $a, b_{1}, \ldots, b_{k}$ be as in Theorem 2.10. Then $P(\phi)$ is simple if and only if

(26) $H(\phi)$ is contained in the algebra generated by 1 and $a^{p}, n-k=m p$,

(27) $\phi\left(a^{n-k-1}, a\right)=\sum_{i=0}^{m-1} \alpha_{i p} a^{i p}, \alpha_{i p}$ in $F, i=0, \cdots, m-1$, with $\alpha_{0} \neq 0$.

(28) $\phi\left(b_{i}, b_{j}\right)=x_{i, j}, i, j=1, \cdots, l$, where $X=\left(x_{i, j}\right)$ is the matrix of Theorem 2.6, $l=k$ if $k$ is even and $l=k-1$ if $k$ is odd. Furthermore, if $k$ is odd and $\phi\left(b_{k}, b_{j}\right)=0$ for all $j$, then for any $\alpha, \beta$ in $F, \phi\left(a a^{n-k-1}+\beta b_{k}, a\right) \neq 0$.

Proof. As in the last theorem we note that $\phi\left(a^{i}, a^{j}\right)=0$ if either $p \mid i$ or $p \mid j$ or $p \nmid(i+j)$ or $i+j>n$. Also, since $a^{2} b_{i}=0$, using Theorem 2.3 it follows that $\phi\left(a^{s}, b_{i}^{r}\right)=0$ if either $s>1$ or $r>1, i=1, \cdots, k$. Now suppose $P(\phi)$ is simple. Then $\phi\left(a^{n-k-1}, a\right) \neq 0$. Let $x, y$ be in $P$. Then

$$
\phi(x, y)=\sum_{i=0}^{n-k-1} \gamma_{i} a^{i}+\sum_{j=1}^{k} \delta_{j} b_{j} ; \quad \gamma_{i}, \delta_{j} \text { are in } F .
$$

Since $\phi\left(\phi(x, y), a^{n-k-1}\right)$ is an antiflexible map, we have $0=\phi\left(\phi(x, y), a^{n-k-1}\right)$ $=\gamma_{1} \phi\left(a, a^{n-k-1}\right)$ which implies that $\gamma_{1}=0$. Suppose that $\gamma_{i}=0$ for all $i<l$ with $p \nmid i$, $p \nmid l$ and $n-k-l>1$. Then

$$
0=\phi\left(\phi(x, y), a^{n-k-l}\right)=-l \gamma_{l} \phi\left(a^{n-k-l}, a\right) .
$$

Therefore, $\gamma_{l}=0$. Thus by mathematical induction $\gamma_{i}=0$ for $1 \leq i \leq n-k-2$ with $p .\left\{i\right.$ and we have $\phi(x, y)=\sum_{i=0}^{m-1} \gamma_{i p} a^{i p}+Y_{1}$ where $Y_{1}=\gamma_{n-k-1} a^{n-k-1}$ $+\Sigma_{j=1}^{k} \delta_{j} b_{j}$ is in $M$. For any $z$ in $P, 0=\phi(\phi(x, y), z)=\phi\left(Y_{1}, z\right)$, so $Y_{1}=0$. Consequently, (26) is satisfied.

On taking $x=a^{n-k-1}, y=a$, we get

$$
\phi\left(a^{n-k-1}, a\right)=\sum_{i=0}^{m-1} \alpha_{i p} a^{i p} ; \quad \alpha_{i p} \text { is in } F .
$$

If $a_{0}=0$ then $a$ generates a proper ideal of $P(\phi)$. So $a_{0} \neq 0$ and we have (27). The first part of (28) follows from Theorem 2.7. Now if $k$ is odd, $\phi\left(b_{k}, b_{j}\right)=0$ for $1 \leq j \leq k$, and if $\alpha$ and $\beta$ are arbitrary elements of $F$, then $\phi\left(a a^{n-k-1}+\beta b_{k}, b_{i}\right)=0$ 
for $1 \leq i \leq k$. Since $a a^{n-k-1}+\beta b_{k}$ is in $M, \phi\left(\alpha a^{n-k-1}+\beta b_{k}, a\right) \neq 0$.

Conversely, suppose $\phi$ satisfies (26), (27) and (28) and $J$ is an ideal of $P(\phi)$. Let $x$ be in $J, x \neq 0$. Then

$$
x=\sum_{i=0}^{n-k-1} \gamma_{i} a^{i}+\sum_{j=1}^{k} \delta_{j} b_{j} ; \quad \gamma_{i}, \delta_{j} \text { are in } F .
$$

Since $x \neq 0$, at least one of the $\gamma_{i}$ or one of the $\delta_{j}$ is different from zero. Thus we have two cases:

Case 1. Suppose $\gamma_{i} \neq 0$ for some $i, 1 \leq i \leq n-k-2$. Let $j$ be the least integer such that $\gamma_{i} \neq 0$. If $p \nmid j$ then since $n-k-j>1, \phi\left(x, a^{n-k-j}\right)=$ $\gamma_{j} \phi\left(a^{j}, a^{n-k-j}\right)=-j \gamma_{j}\left(\sum_{i=0}^{m-1} \alpha_{i p} a^{i p}\right)$. Therefore $\left(-1 / j \gamma_{j} \alpha_{0}\right) \phi\left(x, a^{n-k-j}\right)=1-z$ is in $J$ with $z$ nil. Similarly if $p \mid j$ then $\left(-1 /(j+1) \gamma_{j} a_{0}\right) \phi\left(x * a, a^{n-k-j-1}\right)=1-z$ is in $J$ with $z$ nil. Thus, in either case, for some positive integer $r$ we have $1=(1-z)\left(1+z+\cdots+z^{r-1}\right)$ is in $J$. Hence $J=P(\phi)$ and so $P(\phi)$ is simple.

Case 2. Suppose $\gamma_{i}=0$ for $0 \leq i \leq n-k-2$ and $\delta_{j} \neq 0$ for some $j$, say $j=$ $l$. If $l<k$, then by Theorem 2.7 there is a $j$ such that $\phi\left(b_{l}, b_{j}\right)=x_{l, j}$ is in $J \cap F$ and $x_{l, j} \neq 0$. Hence 1 is in $J$ and so $J=P(\phi)$. On the other hand, if $\delta_{k} \neq 0$ then if $k$ is even then there is a $j$ such that $\phi\left(b_{k}, b_{j}\right)=x_{l, j}$ is in $J \cap F, x_{l, j} \neq 0$ and so $J=P(\phi)$. If $k$ is odd and if $\phi\left(b_{k}, b_{j}\right) \neq 0$ for some $j$ then as in Case 1 it can be shown that $J=P(\phi)$. Therefore, let us now assume that $k$ is odd and $\phi\left(b_{k}, b_{j}\right)=0$ for all $j=1, \cdots, k$. Then since $x=\gamma_{n-k-1} a^{n-k-1}+\delta_{k} b_{k}$, by (28), $\phi(x, a) \neq 0$ and once again it can be shown that $J=P(\phi)$. Hence $P(\phi)$ is simple and the proof is complete.

Having determined all nodal simple antiflexible algebras of types $(n, n)$ and $(n-k, n, k+1)$, our next interest is those of type $(n-2, n) . \quad$ If $\operatorname{dim} \overline{N_{1}}=2$ and $\operatorname{dim} \overline{N_{2}}$ $=1$ then $\operatorname{dim} \overline{N_{i}}=1$ for all $2 \leq i \leq n-3$ so that $\operatorname{dim} N=n-2$. Since $\operatorname{dim} N=n-1$ we conclude that either $\operatorname{dim} \bar{N}_{1}=3$ and $\operatorname{dim} \bar{N}_{i}=1$ for $2 \leq i \leq n-3$ or $\operatorname{dim} \overline{N_{1}}=2$, $\operatorname{dim} \overline{N_{2}}=2$ and $\operatorname{dim} N_{i}=1$ for $3 \leq i \leq n-3$. We have proved the following lemma.

Lemma 3.1. If $N$ is of type $(n-2, n)$ then $N$ is either of the type $(n-2, n, 3)$ or of the type $(n-2,2,2)$.

We have determined all simple nodal totally antiflexible algebras of type $(n-2, n, 3)$. So now we will be interested in algebras of type $(n-2, n, 2,2)$ and in general of type $(n-k, n, k, 2)$.

Theorem 3.3. Let $N$ be an associative, commutative nilalgebra of type $(n-k, n, k, 2)$ over a field of char. $\neq 2,3$. Then there exist $a, b, c, i=1, \ldots$, $k-1$, sucb that $b_{i}$ is in $N_{1}^{\prime}, c$ is in $N_{2}^{\prime}$ and $\left\{a, \cdots, a^{n-k-1}, b_{1}, \cdots, b_{k-1}, c\right\}$ is a basis of $N$ with $a^{2} b_{i}=0, a b_{i} b_{j}=\beta_{i, j} a^{n-k-1}, b_{i} b_{j} b_{l}=\gamma_{i, j, l} a^{n-k-1}, i, j$, $l=1, \cdots, k-1$. Furthermore, $c$ can be chosen to be either $a b_{1}$ or $b_{1}^{2}$ or $b_{1} b_{2}$. 
Proof. By Theorem 2.8, since $\operatorname{dim} \overline{N_{3}}=1$, there is an element $a$ in $N$ with $a^{n-k-1} \neq 0$. Let $c_{1}, \cdots, c_{k-1}, c$ be chosen so that $c_{1}, \cdots, c_{k-1}$ are in $N_{1}^{\prime}$, $c$ in $N_{2}^{\prime}$ and $\left\{a, \ldots, a^{n-k-1}, c_{1}, \ldots, c_{k-1}, c\right\}$ is a basis for $N$. (This is possible since $\operatorname{dim} \overline{N_{1}}=k$ and $\operatorname{dim} \overline{N_{2}}=2$.) Then $N_{3}$ is spanned by $a^{3}, \cdots, a^{n-k-1}$, so

$$
a^{2} c_{i}=\sum_{j=3}^{n-k-1} a_{i, j} a^{j}=a^{2} \sum_{j=3}^{n-k-1} \alpha_{i, j} a^{j-2} ; \quad i=1, \cdots, k-1 .
$$

Define $b_{i}=c_{i}-\sum_{j=3}^{n-k-1} \alpha_{i, j} a^{j-2} ; i=1, \ldots, k-1$. Clearly $a^{2} b_{i}=0, i=1$, $\cdots, k-1$, and $\left\{a, \cdots, a^{n-k-1}, b_{1}, \cdots, b_{k-1}, c\right\}$ is a basis of $N$ with $b_{i}$ in $N_{1}^{\prime}$. Since $a b_{i} b_{j}$ is in $N_{3}, a b_{i} b_{j}=\sum_{l=3}^{n-k-1} \beta_{i, j, l} a^{l}$. Then we have $0=a^{2} b_{i} b_{j}$ $=\Sigma_{l=3}^{n-k-2} \beta_{i, j, l^{a}}$ and so $\beta_{i, j, l}=0$ for $l=3, \ldots, n-k-2 ; i, j=1, \ldots, k-1$. Defining $\beta_{i, j}=\beta_{i, j, n-k-1}$ one gets $a b_{i} b_{j}=\beta_{i, j} a^{n-k-1} ; i, j=1, \cdots, k-1$.

Also, $a b_{i} b_{j} b_{l}=\beta_{i, j} a^{n-k-1} b_{l}=0$ (since $n-k \geq 3$ ); $i, j, l=1, \ldots, k-1$. Now $b_{i} b_{j} b_{l}$ is in $N_{3}$ so $b_{i} b_{j} b_{l}=\sum_{t=3}^{n-k-1} a^{t}$ and $0=a b_{i} b_{j} b_{l}=\sum_{t=3}^{n-k-2} \gamma_{i, j, l, t} a^{t+1}$. Hence $\gamma_{i, j, l, t}=0$ for $i, j, l=1, \ldots, k-1$ and $t=3, \cdots, n-k-2$. Defining $\gamma_{i, j, l}=\gamma_{i, j, l, n-k-1}$, we have $b_{i} b_{j} b_{l}=\gamma_{i, j, l} a^{n-k-1}$.

To prove the second part of the theorem, we only need to show that at least one of $a b_{1}, b_{1}^{2}, b_{1} b_{2}$ is not in $N_{3}$. If either $a b_{i}$ or $b_{j}^{2}$ or $b_{i} b_{j}$ is not in $N_{3}$ for some $i$ or $j$ then one can rearrange the $b_{i}$ 's and the proof will be complete.

Hence assume that $a b_{i}, b_{i} b_{j}$ are in $N_{3}$ for all $i, j=1, \ldots, k-1$. Since $c$ is in $N_{2}^{\prime}$, we have

$$
\begin{aligned}
c & =\left(\alpha a+\sum_{i=1}^{k-1} \alpha_{i} b_{i}\right)\left(\beta a+\sum_{j=1}^{k-1} \beta_{j} b_{j}\right)+z ; \quad z \text { in } N_{3} ; \\
& =\alpha \beta a^{2}+z^{\prime} ; \quad z^{\prime} \text { in } N_{3} .
\end{aligned}
$$

Therefore, $c, a^{2}, a^{3}, \cdots, a^{n-k-1}$ are linearly dependent which is impossible and we are done.

Lemma 3.2. Let $\phi$ be an antiflexible map on an associative commutative algebra $P$ of char. $\neq 2,3$ with $a^{2} b=0$. Then

(29) $\phi\left(a b^{2}, a^{s}\right)=0$ for $s \geq 0$,

(30) $\phi\left(a^{s}, b\right)=0$ for $s>2$,

(31) $\phi\left(a b, a^{s}\right)=0$ for $s>1$,

(32) $\phi\left(a^{r}, b^{s}\right)=0$ for $r>1$ and $s>1$,

(33) $2 \phi(a b, a)+\phi\left(a^{2}, b\right)=0$,

(34) $2 \phi(a b, b)+\phi\left(b^{2}, a\right)=0$.

Proof. (29) is obvious for $s=0$. If $s>0$, consider $a b, b, a^{s}$, then, by Theorem 2.3, $\phi\left(a b^{2}, a^{s}\right)+\phi\left(a^{s} b, a b\right)+\phi\left(a^{s+1} b, b\right)=0$. Since $a^{s} b=0$ for $s>1$ 
and for $s=1, \phi(a b, a b)=0, \phi\left(a^{s} b, a b\right)=0=\phi\left(a^{s+1} b, b\right)$. Therefore $\phi\left(a b^{2}, a^{s}\right)$ $=0$. To prove (30) let $s>2$ and consider $a_{1}, \cdots, a_{s}, b$ where $a_{i}=a$ for $i=1$, $\cdots, s$. Then using Theorem 2.3 we have $\phi\left(a^{s}, b\right)+s \phi\left(a^{s-1} b, a\right)=0$ which implies that $\phi\left(a^{s}, b\right)=0$. Similarly, to prove (31) consider $a, b, a^{s}$ and apply Theorem 2.3.

Now suppose $r=2$ and $s>1$. Consider $a^{2}, b_{1}, \cdots, b_{s}$ with $b_{i}=b$ for $i=$ $1, \cdots, s$. Then by Theorem 2.3 we have $\phi\left(b^{s}, a^{2}\right)+s \phi\left(a^{2} b^{s-1}, b\right)=0$. Since $a^{2} b^{s-1}=0, \phi\left(a^{2}, b^{s}\right)=0$. If $r>2$, consider $a_{1}, \cdots, a_{r^{\prime}} b^{s}$ where $a_{i}=a$ for all $i=1, \cdots, r$, then using Theorem $2.3, \phi\left(a^{r}, b^{s}\right)+r \phi\left(a^{r-1} b^{s}, a\right)=0$ which implies $\phi\left(a^{r}, b^{s}\right)=0$. Considering $a, a, b$ and applying Theorem 2.3 we get (33), and (34) follows immediately by using Theorem 2.3 for $a, b, b$.

We are now ready to prove the following theorem.

Theorem 3.4. Let $P=F \cdot 1 \oplus N$ where $N$ is an associative commutative algebra of type $(n-k, n, k, 2)$ with $n-k>3$ over a field $F$ of char. $\neq 2,3$. If $P$ is nearly simple then $N$ is spanned by $a, \ldots, a^{n-k-1}, b_{1}, \cdots, b_{k-1}, c$ where $a^{2} b_{i}=a b_{i} b_{j}=b_{i} b_{j} b_{l}=0, i, j, l=1, \ldots, k-1, c$, is either $a b_{1}$ or $b_{1}^{2}$ or $b_{1} b_{2}$ and $n-k=m$ char $F$ for $m>0$.

Proof. By Theorem 3.3, there are elements $a, b_{1}, \ldots, b_{k-1}, c$ with $N$ spanned by $a, \cdots, a^{n-k-1}, b_{1}, \cdots, b_{k-1}, c$. Furthermore, $a^{2} b_{i}=0, a b_{i} b_{j}=$ $\beta_{i, j} a^{n-k-1}, b_{i} b_{j} b_{l}=\gamma_{i, j, l^{n-k-1}}$ for $i, j, l=1, \cdots, k-1$ and $c$ is either $a b_{1}$ or $b_{1}^{2}$ or $b_{1} b_{2}$. From this it is clear that $M$ is a subspace of the space spanned by $\left\{a^{n-k-1}, a b_{i}, b_{i} b_{j} ; i, j=1, \cdots, k-1\right\}$.

Assume $P$ is nearly simple. Then there is a $\phi$ with $P(\phi)$ simple. We first show that for every $i$ and $j, a b_{i}$ and $b_{i} b_{j}$ are in $M$. To do this it is necessary ind sufficient to prove that each $\beta_{i, j}=0$ and each $\gamma_{i, j, l}=0$. If $x \neq 0$ is in $M$, Theorem 2.5 assures the existence of a $y$ in $N$ with $\phi(x, y) \neq 0$. Thus, if $x$ in $M$ has the property that $\phi(x, y)=0$ for all $y$ in $N$ then $x=0$. Since $n-k-1>$ 2 by (28), $\phi\left(a^{n-k-1}, b_{i}\right)=0$ for all $i$. Hence $\phi\left(a b_{i} b_{j}, b_{l}\right)=\beta_{i, j} \phi\left(a^{n-k-1}, b_{l}\right)=$ 0 and $\phi\left(b_{i} b_{j} b_{l}, b_{b}\right)=\gamma_{i, j, l} \phi\left(a^{n-k-1}, b_{b}\right)=0$ for all $b, i, j, l=1, \cdots, k-1$. Also by Theorem 2.3, $\phi\left(a b_{i} b_{j}, a\right)=-\phi\left(a^{2} b_{i}, b_{j}\right)-\phi\left(a^{2} b_{j}, b_{i}\right)=0$ and $\phi\left(b_{i} b_{j} b_{l}, a\right)$ $=-\phi\left(a b_{i} b_{j}, b_{l}\right)-\phi\left(a b_{j} b_{l}, b_{i}\right)-\phi\left(a b_{i} b_{l}, b_{j}\right)=0$. Since $a b_{i} b_{j}, b_{i} b_{j} b_{l}$ are in $M, a b_{i} b_{j}=$ $b_{i} b_{j} b_{l}=0$ for $i, j, l=1, \cdots, k-1$. Thus we have shown that $M$ is the space spanned by $\left\{a^{n-k-1}, a b_{i}, b_{i} b_{j} ; i, j=1, \cdots, k-1\right\}$.

Since $a^{n-k^{2}-1} \neq 0$ is in $M$ there is a $y$ in $N$ with $\phi\left(a^{n-k-1}, y\right) \neq 0$. For $q>1, a^{q}$ is in $N_{2}$ and $\phi\left(a^{n-k-1}, b_{i}\right)=0$ for $i=1, \ldots, k-1$; so we conclude $\phi\left(a^{n-k-1}, a\right) \neq 0$. But by Theorem $2.3,(n-k) \phi\left(a^{n-k-1}, a\right)=0$, so $n-k=$ $m$ char $F$ for some $m>0$.

As an immediate corollary, we have 
Corollary 3.1. Let $P=F \cdot 1 \oplus N$ where $N$ is an associative commutative nilalgebra of type $(n-2, n, 2,2)$ with $n-2>2$ over a field $F$ of char. $\neq 2,3$. Then there are elements $a, b, c$ such that $b$ is in $N_{1}^{\prime}, c$ is in $N_{2}^{6}$, and $N$ is spanned by $a, \cdots, a^{n-3}, b, c$ with $a^{2} b=0, a b^{2}=\beta a^{n-3}, b^{3}=\gamma a^{n-3}$ and $c$ is either $a b$ or $b^{2}$. Furthermore, if $P$ is nearly simple and $n-2>3$, then $\beta=0=\gamma$ and $n-2=m$ char $F$ for some $m>1$.

Theorem 3.5. Let $P=F \cdot 1 \oplus N$ be an associative commutative nearly nodal algebra of type $(n-2, n, 2,2)$ with $n-2>3$ over a field $F$ of char. $\neq 2,3$. Then $P$ is nearly simple if and only if

(35) $N$ is spanned by $\left\{a, \cdots, a^{n-3}, b, a b\right\}$ with $a^{2} b=a b^{2}=b^{3}=0$ and $b^{2}=$ $a a^{n-3}$ for some a in $F$,

(36) $n-2=m$ char $F$ for some positive integer $m$ and if $b^{2}=0$ then $m>1$.

Proof. Suppose $P$ is nearly simple. Then there is an antiflexible map $\phi$ with $P(\phi)$ simple. To prove (35) we only need to show that $c=a b$ and $b^{2}=a a^{n-3}$ in Corollary 3.1. If $c=b^{2}$ then $a b=\sum_{i=2}^{n-3} \alpha_{i} a^{i}+\beta b^{2}$ and so $0=a^{2} b=\sum_{i=2}^{n-4} \alpha_{i} a^{i+1}$. Therefore, $a_{i}=0$ for $i=2, \cdots, n-4$ and $a b=a_{n-3} a^{n-3}+\beta b^{2}$. Thus $\phi(a b, b)$ $=a_{n-3} \phi\left(a^{n-3}, b\right)+\beta \phi\left(b^{2}, b\right)=0$. Consequently, by Lemma 3.2(34), $\phi\left(b^{2}, a\right)=0$. Since $b^{2}$ is in $M, b^{2}=0$ which is impossible. Hence $c=a b$ and $b^{2}=\sum_{i=2}^{n-3} \beta_{i} a^{i}$ $+\gamma a b$. Now $0=a b^{2}=\sum_{i=2}^{n-4} \beta_{i} a^{i+1}$, so $\beta_{i}=0$ for $i=2, \cdots, n-4$. Thus $b^{2}=$ $\beta_{n-3} a^{n-3}+\gamma a b$ and $0=\phi\left(b^{2}, b\right)=\beta_{n-3} \phi\left(a^{n-3}, b\right)+\gamma \phi(a b, b)=\gamma \phi(a b, b)$. If $\phi(a b, b)=0$ then, by Lemma 3.2(34), $\phi\left(b^{2}, a\right)=0$ and consequently $b^{2}=0=$ $0 \cdot a^{n-3}$. On the other hand, if $\phi(a b, b) \neq 0$, then $\gamma=0$ and defining $a=\beta_{n-3}$ we have $b^{2}=a \cdot a^{n-3}$.

By Corollary 3.1, $n-2=m$ char $F$ for some $m \geq 1$. Suppose that $b^{2}=0$ and $m=1$. Then it is easy to show that $H(\phi) \subseteq F \cdot 1$. Since $b^{2}=0$, by Lemma 3.2(34), $\phi(a b, b)=0$. Therefore, since $a b \neq 0$ is in $M, \phi(a b, a)=\beta \neq 0$. Let $\phi\left(a^{n-3}, a\right)$ $=\delta, \delta \neq 0$. Define $x=\beta a^{n-3}-\delta a b$, then $x \neq 0, x$ is in $M$ and $\phi(x, z)=0$ for all $z$ in $N$. This is a contradiction. Hence $m>1$.

Conversely, suppose $P$ satisfies (35) and (36) and $b^{2} \neq 0$. Define $\phi$ on the basis of $P$ as follows:

$$
\begin{aligned}
& \phi\left(a^{i}, a^{j}\right)= \begin{cases}0 & \text { if } i+j \neq n-2, \\
j & \text { if } i+j=n-2\end{cases} \\
& \phi\left(b^{2}, a\right)=2=-\phi\left(a, b^{2}\right) ; \\
& \phi(a b, b)=-1=-\phi(b, a b) ; \\
& \phi(x, y)=0 \text { for any other pair of basis elements } x \text { and } y .
\end{aligned}
$$

Extend $\phi$ bilinearly to $P \times P$. Then it is easy to verify that $\phi$ is an antiflexible map and that (17) and (18) are satisfied. Hence, by Theorem 2.5, $P(\phi)$ is simple. 
If $b^{2}=0$, then by (36), $m>1$ so $a^{p} \neq 0$. Define $\phi$ on the basis of $P$ as follows:

$$
\begin{aligned}
\phi\left(a^{i}, a^{j}\right) & = \begin{cases}0 & \text { if } i+j \neq n-2 \\
j & \text { if } i+j=n-2\end{cases} \\
\phi(a b, a) & =a^{p}=-\phi(a, a b) \\
\phi\left(a^{2}, b\right) & =-2 a^{p}=-\phi\left(b, a^{2}\right) ; \\
\phi(x, y) & =0 \text { for any other pair of basis elements } x \text { and } y .
\end{aligned}
$$

Extend $\phi$ bilinearly to $P \times P$, then it is routine to verify that $\phi$ is an antiflexible map and (17) and (18) hold. Therefore, by Theorem 2.5, $P(\phi)$ is simple.

Having determined nearly simple algebras of type $(n-2, n)$ our next interest is to find all possible antiflexible maps that give rise to simple antiflexible algebras of type $(n-2, n)$.

Theorem 3.6. Let $P=F \cdot 1 \oplus N$ be an associative commutative nearly simple, nearly nodal algebra of type $(n-2, n, 2,2)$ with $n-2>3$ over a field $F$ of characteristic $p \neq 2,3$ and let $\phi$ be an antiflexible map on $P$. If $a$ and $b$ are as in Theorem 3.5, then $P(\phi)$ is simple if and only if

(37) $H(\phi)$ is a subset of the algebra generated by 1 and $a^{p}, n-2=m p$,

(38) $\phi\left(a^{n-3}, a\right)=\sum_{i=0}^{m-1} a_{i p} a^{i p}, \alpha_{i p}$ in $F$ for $i=0, \cdots, m-1$ with $\alpha_{0} \neq 0$,

(39) if $b^{2}=0$, then $\phi(a b, a)=\sum_{i=1}^{m-1} \gamma_{i p} a^{i p} \neq 0$ and, for any $\delta$ in $F, \phi(a b, a)$ $\neq \delta \phi\left(a^{n-1}, a\right)$.

Proof. Since $P$ is nearly simple (35) and (36) of Theorem 3.5 hold.

Assume $P(\phi)$ is simple. By Theorem 2.3, $\phi\left(a^{i}, a^{j}\right)=-i \phi\left(a^{i+j-1}, a\right)=j \phi\left(a^{i+j-1}, a\right)$, so $\phi\left(a^{i}, a^{j}\right)=0$ if $p \mid i$ or $p \mid j$ or $p \nmid(i+j)$ or $i+j>n-2$. Since $a^{n-3}$ is in $M$ and $\phi\left(a^{n-3}, b\right)=0$ (by Lemma 3.2), $\phi\left(a^{n-3}, a\right) \neq 0$. Let $x, y$ be in $P$, then

$$
\phi(x, y)=\sum_{j=0}^{n-3} \beta_{i} a^{i}+\beta b+\gamma a b, \quad \beta, \gamma, \beta_{i} \text { in } F \text { for } i=1, \cdots, n-3 \text {. }
$$

Now, $0=\phi\left(\phi(x, y), a^{n-3}\right)=\beta_{1} \phi\left(a, a^{n-3}\right)$ which implies $\beta_{1}=0$. Suppose $\beta_{i}$ $=0$ for all $i<k$ with $p \nmid i, p \nmid k$ and $k<n-3$. Then we have

$$
0=\phi\left(\phi(x, y), a^{n-k-2}\right)=\alpha_{k} \phi\left(a^{k}, a^{n-k-2}\right)=-k \alpha_{k} \phi\left(a^{n-3}, a\right) .
$$

Therefore, $a_{k}=0$ and hence, by mathematical induction, $a_{i}=0$ for all $i<n-3$ with $p \nmid i$. Thus $\phi(x, y)=\sum_{i=0}^{m-1} \beta_{i p} a^{i p}+\beta b+Y$ where $Y=\beta_{n-3} a^{n-3}+\gamma a b$ is in $M$. If $b^{2} \neq 0$, then by Lemma $3.2, \phi(a b, b) \neq 0$ and $0=\phi(\phi(x, y), a b)=$ $\beta \phi(b, a b)$. Consequently, $\beta=0$. On the other hand, if $b^{2}=0$ then $\phi(a b, a) \neq 0$ and so $\phi\left(a^{2}, b\right) \neq 0$ (by Lemma 3.2). Since $a^{2}$ is in $N_{2}$, we have $0=\phi\left(\phi(x, y), a^{2}\right.$ ) $=\beta \phi\left(b, a^{2}\right)$ which implies $\beta=0$. Now for any $z$ in $P, 0=\phi(\phi(x, y), z)=\phi(Y, z)$, so $Y=0$ and we have (37). 
Let $x=a^{n-3}, y=a$, then we have $\phi\left(a^{n-3}, a\right)=\sum_{i=0}^{m-1} a_{i p} a^{i p}$. If $\alpha_{0}=0$ then $a$ generates a proper ideal of $P(\phi)$, so $\alpha_{0} \neq 0$. Now suppose $b^{2}=0$, then $\phi\left(b^{2}, a\right)=0=\phi(a b, b)$. Since $a b$ is in $M, \phi(a b, a) \neq 0$. If there is a $\delta$ in $F$ with $\phi(a b, a)=\delta \phi\left(a^{n-3}, a\right)$, define $x=a b-\delta a^{n-3}$. Then $x$ is in $M$ and $\phi(x, z)=0$ for all $z$ in. $P$ which is a contradiction. Hence for any $\delta$ in $F, \phi(a b, a) \neq$ $\delta \phi\left(a^{n-3}, a\right)$.

Conversely, suppose $\phi$ satisfies (37), (38), and (39). We first note that $M$ is spanned by $\left\{a^{n-3}, a b\right\}$. Let $x$ be a nonzero element in $M$. Then $x=\beta a^{n-3}+$ $\gamma a b, \beta, \gamma$ in $F$, such that either $\beta \neq 0$ or $\gamma \neq 0$. By Theorem $3.5, b^{2}=a a^{n-3}$ for some $\alpha$ in $F$. Now suppose $\gamma \neq 0$. If $\alpha \neq 0$, then $\phi(x, b)=\beta \phi\left(a^{n-3}, b\right)+$ $\gamma \phi(a b, b)=(-1 / 2) y \alpha \phi\left(a^{n-3}, a\right) \neq 0$, and if $\alpha=0$ then, by (39), $\phi(x, a)=\beta \phi\left(a^{n-3}, a\right)+$ $\gamma \phi(a b, a) \neq 0$. On the other hand, if $\gamma=0$, then $x=\beta a^{n-3}$, so $\phi(x, a)=\beta$ . $\phi\left(a^{n-3}, a\right) \neq 0$. Thus $(17)$ is satisfied. Also, it is easy to verify that (18) holds. Hence by Theorem $2.5, P(\phi)$ is simple.

Thus we have determined all nodal simple totally antiflexible algebras of type $(n-2, n)$ with $n-2>3$. The case $n-2=3$ will be solved in $\S$ IV of this paper. In the rest of this section we will try to generalize some of the results obtained so far.

Theorem 3.7. Let $N$ be an associative commutative nilalgebra of type $\left(m, n, d_{1}, d_{2}\right)$ over a field of char. $\neq 2,3$. Then there exists $a, b_{i}, c_{j}$ $\left(i=1, \cdots, d_{1}-1 ; j=1, \ldots, d_{2}-1\right)$ such that $b_{i}$ is in $N_{1}^{\prime}, c_{j}$ in $N_{2}^{\prime}$ and $\left\{a, \cdots, a^{m-1}, b_{i}, c_{j} ; i=1, \ldots, d_{1}-1 ; j=1, \ldots, d_{2}-1\right\}$ is a basis of $N$ with

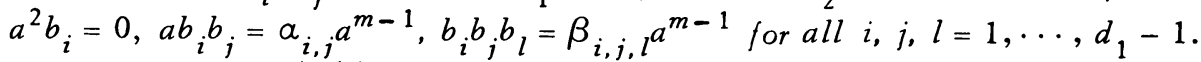
Furthermore, $d_{2} \leq(1 / 2) d_{1}\left(d_{1}+1\right), c_{i}$ is in $A$ for all $i$ where $A=\left\{a b_{i}, b_{i} b_{j}: i\right.$, $\left.j=1, \cdots, d_{1}-1\right\}$, and if $d_{2}=(1 / 2) d_{1}\left(d_{1}+1\right)$ then $A \cap N_{3}$ is a null set.

Proof. Since $\operatorname{dim} \bar{N}_{3}=1$, by Theorem 2.8 there is an $a$ in $N$ with $a^{m-1} \neq 0$. Also since $N$ is of type $\left(m, n, d_{1}, d_{2}\right)$ there exists $a, g_{i}, c_{j}\left(i=1, \ldots, d_{1}-1 ; j=1, \ldots\right.$, $\left.d_{2}-1\right)$ such that $g_{i}$ is in $N_{1}^{\prime}, c_{j}$ is in $N_{2}^{\prime}$ and $\left\{a, \cdots, a^{m-1}, g_{i^{\prime}}, c_{j}: i=1, \ldots, d_{1}-1\right.$; $\left.j=1, \ldots, d_{2}-1\right\}$ is a basis of $N$. Then $N_{3}$ is spanned by $a^{3}, \cdots, a^{m-1}$ and so

$$
a^{2} g_{i}=\sum_{j=3}^{m-1} \gamma_{i, j} a^{j}=a^{2} \sum_{j=3}^{m-1} \gamma_{i, j} a^{j-2}, \quad \gamma_{i, j} \text { in } F \text {. }
$$

Defining $b_{i}=g_{i}-\sum_{j=3}^{m-1} \gamma_{i, j} a^{j-2}$ we get that $\left\{a, \cdots, a^{m-1}, b_{i}, c_{j}: i=1, \cdots, d_{1}-1 ; j=\right.$ $\left.1, \ldots, d_{2}-1\right\}$ is a basis of $N$ with $a^{2} b_{i}=0$ for all $i$. Since $a b_{i} b_{j}$ is in $N_{3}, a b_{i} b_{j}=$ $\sum_{t=3}^{m-1} a_{i, j, t} a^{t}$. Then $0=a^{2} b_{i} b_{j}=\sum_{t=3}^{m-2} \alpha_{i, j, t} a^{t+1}$ which implies that $\alpha_{i, j, t}=0$ for $i, j=$ $1, \cdots, d_{1}-1 ; t=3, \cdots, m-2$. Hence defining $\alpha_{i, j}=\alpha_{i, j, m-1}$ one gets $a b_{i} b_{j}=$ $a_{i, j} a^{m-1}$ for $i, j=1, \ldots, d_{1}-1$. Since $m \geq 3, a b_{i} b_{j} b_{l}=a_{i, j} a^{m-1} b_{l}=0$. Since $b_{i} b_{j} b_{l}$ is in $N_{3}, b_{i} b_{j} b_{l}=\Sigma_{t=3}^{m-1} \beta_{i, j, l, t} a^{t}$. Then $0=a b_{i} b_{j} b_{l}=\sum_{t=3}^{m-2} \beta_{i, j, l, t} a^{t+1}$ which implies $\beta_{i, j, l, t}=0$ for $i, j, l=1, \ldots, d_{1}-1 ; t=3, \cdots, m-2$. Therefore, 
defining $\beta_{i, j, l}=\beta_{i, j, l, m-1}$, we get $b_{i} b_{j} b_{l}=\beta_{i, j, l^{a^{m-1}}}$ for $i, j, l=1, \cdots, d_{1}-1$.

To prove the second part of this theorem we note that the number of ways of picking two distinct elements out of $d_{1}$ elements is $(1 / 2) d_{1}\left(d_{1}-1\right)$ and $b_{1}^{2}, \ldots$, $b_{d_{1-1}-1}^{2}$ are in $A$. Therefore, the cardinality of $A=(1 / 2) d_{1}\left(d_{1}-1\right)+d_{1}+1=$ $(1 / 2) d_{1}\left(d_{1}+1\right)-1$. Since $N_{2}$ is contained in the space spanned by $A \cup\left\{a^{2}, \ldots\right.$, $\left.a^{m-1}\right\}, \operatorname{dim} N_{2} \leq(1 / 2) d_{1}\left(d_{1}+1\right)-1+m-2$. But $\operatorname{dim} N_{2}=d_{2}+m-3$, so $d_{2} \leq$ $(1 / 2) d_{1}\left(d_{1}+1\right)$. Also, since $\operatorname{dim} N_{2}=d_{2}+m-3, A$ has $d_{2}-1$ linearly independent elements. Assume, there are only $t$ linearly independent elements in $A$ that are not in $N_{3}, t<d_{2}-1$. We can choose $c_{1}, \cdots, c_{t}$ to be these elements. Since $c_{t+1}$ is in $N_{2}^{\prime}$,

$$
\begin{aligned}
c_{t+1} & =\left(\alpha a+\sum \alpha_{i} b_{i}\right)\left(\beta a+\sum \beta_{i} b_{i}\right)+z, \quad z \text { in } N_{3}, \\
& =\alpha \beta a^{2}+\sum_{i=1}^{t} \gamma_{i} c_{i}+z^{\prime}, \quad z^{\prime} \text { in } N_{3} .
\end{aligned}
$$

Therefore, $c_{1}, \ldots, c_{t+1}, a^{2}, \ldots, a^{m-1}$ are linearly dependent, which is impossible. Hence $A$ has $d_{2}-1$ linearly independent elements that are not in $N_{3}$ and so $c_{i}$ can be chosen in $A$ for $i=1, \ldots, d_{2}-1$. If $d_{2}=(1 / 2) d_{1}\left(d_{1}+1\right)$ then $\operatorname{dim} N_{2}=$ $d_{2}+m-3=(1 / 2) d_{1}\left(d_{1}+1\right)-1+m-2=$ cardinality of $A+m-2$. Therefore $A \cup\left\{a^{2}, \cdots, a^{m-1}\right\}$ is a basis of $N_{2}$ and hence $A \cap N_{3}$ is a null set.

Theorem 3.8. Let $N$ be an associative commutative nilalgebra of type $\left(m, n, d_{1}, d_{2}, d_{3}\right)$ over a field $F$ of char. $\neq 2,3$. Then there exists $a, b_{i}, c_{i}, f_{k}$ $\left(i=1, \ldots, d_{1}-1 ; j=1, \ldots, d_{2}-1 ; k=1, \ldots, d_{3}-1\right)$ such that $b_{i}$ is in $N_{1}^{\prime}$, $c_{j}$ in $N_{2}^{\prime}, f_{k}$ in $N_{3}^{\prime}$ and $\left\{a, \ldots, a^{m-1}, b_{i}, c_{j}, f_{k} ; i=1, \ldots, d_{1}-1 ; j=1, \ldots\right.$, $\left.d_{2}-1 ; k=1, \ldots, d_{3}-1\right\}$ is a basis of $N$ with $a^{3} b_{i}=0, a^{2} b_{i} b_{j}=a_{i, j} a^{m-1}$.

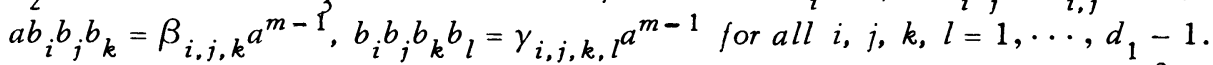
Furthermore, $c_{i}$ is in $A=\left\{a b_{j}, b_{j} b_{k}: j, k=1, \ldots, d_{1}-1\right\} ; f_{k}$ is in $B=\left\{a^{2} b_{i}\right.$, $\left.a b_{i} b_{j}, b_{i} b_{j} b_{l}: i, j, l=1, \ldots, d_{1}-1\right\} ; d_{2} \leq(1 / 2) d_{1}\left(d_{1}+1\right), d_{3} \leq(1 / 6) d_{1}\left(d_{1}+1\right)\left(d_{2}+2\right)$; if $d_{2}=(1 / 2) d_{1}\left(d_{1}+1\right), d_{3}=(1 / 6) d_{1}\left(d_{1}+1\right)\left(d_{1}+2\right)$ then $A \cap N_{3}$ and $B \cap N_{4}$ are null sets respectively.

Proof. Since $\operatorname{dim} \overline{N_{4}}=1$ and char $F \neq 2,3$, by Theorem 2.8 there is an $a$ in $N$ with $a^{m-1} \neq 0$. Also, since $N$ is of type $\left(m, n, d_{1}, d_{2}, d_{3}\right)$ there exists $g_{i}$, $c_{j}, f_{k}\left(i=1, \ldots, d_{1}-1 ; j=1, \ldots, d_{2}-1 ; k=1, \ldots, d_{3}-1\right)$ such that $g_{i}$ is in $N_{1}^{\prime}, c_{j}$ in $N_{2}^{\prime}, f_{k}$ in $N_{3}^{\prime}$ and $N$ is spanned by $\left\{a, \ldots, a^{m-1}, g_{i}, c_{j}, f_{k}: i=1, \ldots\right.$, $\left.d_{1}-1 ; j=1, \cdots, d_{2}-1 ; k=1, \cdots, d_{3}-1\right\}$. Then $N_{4}$ is spanned by $a^{4}, \cdots$, $a^{m-1}$ and so $a^{3} g_{i}=\sum_{j=4}^{m-1} \gamma_{i, j} a^{j}=a^{3} \sum_{j=4}^{m-1} \gamma_{i, j} a^{j-3}$. Defining $b_{i}=g_{i}-\sum_{j=4}^{m-1} \gamma_{i, j} a^{j-3}$ we get that $\left\{a, \cdots, a^{m-1}, b_{i}, c_{j}, f_{k}: i=1, \cdots, d_{1}-1 ; j=1, \cdots, d_{2}-1 ; k=1\right.$, $\left.\cdots, d_{3}-1\right\}$ is a basis of $N$ with $a^{3} b_{i}=0$ for $i=1, \ldots, d_{1}-1$. Since $a^{2} b_{i} b_{j}$ is 
in $N_{4}, a^{2} b_{i} b_{j}=\sum_{t=4}^{m-1} \alpha_{i, j, t} a^{t}$. Then $0=a^{3} b_{i} b_{j}=\sum_{t=4}^{m-2} a_{i, j, t} a^{t+1}$ which implies $a_{i, j, t}=0$ for $i, j=1, \cdots, d_{1}-1 ; t=3, \cdots, m-2$. Hence defining $\alpha_{i, j}=a_{i, j, m-1}$ one gets $a^{2} b_{i} b_{j}=a_{i, j} a^{m-1}$ for $i, j=1, \ldots, d_{1}-1$. Since $m \geq 4, a^{2} b_{i} b_{j} b_{l}=$ $a_{i, j} a^{m-1} b_{l}=0$. Also, since $a b_{i} b_{j} b_{l}$ is in $N_{4}, a b_{i} b_{j} b_{l}=\sum_{t=4}^{m-1} \beta_{i, j, l, t} a^{t}$. Then $0=a^{2} b_{i} b_{j} b_{l}=\sum_{t=4}^{m-2} \beta_{i, j, l, t} a^{t+1}$ which implies $\beta_{i, j, l, t}=0$ for $i, j, l=1, \ldots$, $d_{1}-1 ; t=1, \ldots, m-2$. Defining $\beta_{i, j, l}=\beta_{i, j, l, m-1}$ one gets $a b_{i} b_{j} b_{l}=\beta_{i, j, l} a^{m-1}$. Now $a b_{i} b_{j} b_{k} b_{l}=\beta_{i, j, k} a^{m-1} b_{l}=0$ and $b_{i} b_{j} b_{k} b_{l}=\sum_{t=4}^{m-1} \gamma_{i, j, k, l, t} a^{t}$. So $0=$ $a b_{i} b_{j} b_{k} b_{l}=\sum_{t=4}^{m-2} \gamma_{i, j, k, l, t} a^{t+1}$ which implies $\gamma_{i, j, k, l, t}=0$ for $i, j, k, l=1, \ldots$, $d_{1}-1 ; t=4, \cdots, m-2$. Defining $\gamma_{i, j, k, l}=\gamma_{i, j, k, l, m-1}$ we get $b_{i} b_{j} b_{k} b_{l}=$

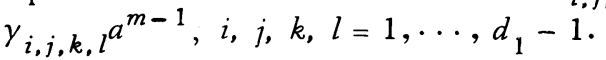

As in the last theorem, $c_{i}$ is in $A, d_{2} \leq(1 / 2) d_{1}\left(d_{1}+1\right)$ and if $d_{2}=$ $(1 / 2) d_{1}\left(d_{1}+1\right)$ then $A \cap N_{3}$ is a null set. Since the number of ways of picking 3 distinct elements out of $d_{1}$ elements is $\left(\begin{array}{c}d_{1} \\ 3\end{array}\right)=(1 / 6) d_{1}\left(d_{1}-1\right)\left(d_{1}-2\right)$ and $d_{1}^{2}$ 1 elements $a^{2} b_{i}, b_{i}^{2} b_{j}, i, j=1, \ldots, d_{1}-1$, are in $B$, cardinality of $B=$ $\left(\begin{array}{c}d_{1} \\ 3\end{array}\right)+d_{1}^{2}-1=(1 / 6) d_{1}\left(d_{1}+1\right)\left(d_{1}+2\right)-1$. Also since $N_{3}$ is contained in the space spanned by $B \cup\left\{a^{3}, \cdots, a^{m-1}\right\}$ and $\operatorname{dim} N_{3}=d_{3}+m-4, d_{3}+m-4 \leq$ $(1 / 6) d_{1}\left(d_{1}+1\right)\left(d_{1}+2\right)-1+m-3$. Therefore, $d_{3} \leq(1 / 6) d_{1}\left(d_{1}+1\right)\left(d_{1}+2\right)$ and $B$ has $d_{3}-1$ linearly independent elements. Assume there are only $t$ linearly independent elements in $B$ that are not in $N_{4}, t<d_{3}-1$. Then we can choose $f_{1}, \cdots, f_{t}$ to be these elements. Since $f_{t+1}$ is $N_{3}^{\prime}$,

$$
\begin{aligned}
f_{t+1} & =\left(\alpha a+\sum \alpha_{i} b_{i}\right)\left(\beta a+\sum \beta_{i} b_{i}\right)\left(\gamma a+\sum \gamma_{i} b_{i}\right)+z, \quad z \text { in } N_{4} \\
& =\alpha \beta \gamma a^{3}+\sum_{i=1}^{t} \delta_{i} f_{i}+z^{\prime}, \quad z^{\prime} \text { in } N_{4} .
\end{aligned}
$$

Therefore, $a^{3}, a^{4}, \ldots, a^{m-1}, f_{1}, \ldots, f_{t+1}$ are linearly dependent, which is a contradiction. Hence $B$ has $d_{3}-1$ linearly independent elements that are not in $N_{4}$. If $d_{3}=(1 / 6) d_{1}\left(d_{1}+1\right)\left(d_{1}+2\right)$, then $B \cup\left\{a^{3}, \ldots, a^{m-1}\right\}$ is a basis of $N_{3}$ so $B \cap N_{4}$ is a null set.

A necessary condition for nodal nearly simple algebras of type $\left(m, n, d_{1}, d_{2}\right)$ is given by the following theorem.

Theorem 3.9. Let $P=F \cdot 1 \oplus N$ where $N$ is an associative commutative nil. algebra of type $\left(m, n, d_{1}, d_{2}\right)$ over a field $F$ of char. $\neq 2,3$. If $P$ is nearly simple and $m>3$ then $a b_{i} b_{j}=b_{i} b_{j} b_{k}=0$ for $i, j, k=1, \ldots, d_{1}-1$, where $a$ and $b_{i}$ are as in Theorem 3.7 and char $F$ divides $m$.

Proof. Suppose $P$ is nearly simple then there is an antiflexible map $\phi$ such that $P(\phi)$ is simple. Since $a^{m-1}$ is in $M, \phi\left(a^{m-1}, b_{i}\right)=0$ (Lemma 3.2) for all $i, \phi\left(a^{m-1}, a\right) \neq 0$. Considering $a, a, b_{i}, b_{j}$ and using Theorem 2.3 we have 
$2 \phi\left(a b_{i} b_{j}, a\right)+\phi\left(a^{2} b_{i}, b_{j}\right)+\phi\left(a^{2} b_{j}, b_{i}\right)=0$. Since $a^{2} b_{i}=0=a^{2} b_{j}, \phi\left(a b_{i} b_{j}, a\right)=$ 0 . Thus $\phi\left(a b_{i} b_{j}, a\right)=\alpha_{i, j} \phi\left(a^{m-1}, a\right)$ (Theorem 3.7) which implies $\alpha_{i, j}=0$ for $i, j=1, \ldots, d_{1}-1$. Similarly it can be shown that $\phi\left(b_{i} b_{j} b_{l}, a\right)=0$ and hence $b_{i} b_{j} b_{l}=0$ for all $i, j, l=1, \ldots, d_{1}-1$. Also, using Theorem 2.3 we have $m \phi\left(a^{m-1}, a\right)=0$, so char $F$ divides $m$.

The following lemma follows from Theorem 2.3.

Lemma 3.3. Let $\phi$ be an antiflexible map on an associative commutative algebra $P$ over a field $F$ of char. $\neq 2,3$ in which $a^{3} b=0$ for some $a, b$ in $P$. Then

(40) $\phi\left(a^{2} b^{2}, a^{s}\right)=0, s \geq 0$,

(41) $\phi\left(a b^{2}, a^{s}\right)=0, s>1$,

(42) $\phi\left(a^{s}, b\right)=0, s>3$,

(43) $\phi\left(a^{2} b, a^{s}\right)=0, s>1$,

(44) $\phi\left(a b, a^{s}\right)=0, s>2$,

(45) $\phi\left(a^{r}, b^{s}\right)=0, r>2$ and $s>1$,

(46) $\phi\left(a^{2} b^{3}, a^{s}\right)=0, s \geq 0$,

(47) $\phi\left(a b^{3}, a^{s}\right)=0, s>1$.

We are now ready to give a necessary condition for nearly simple nodal algebras of type $\left(m, n, d_{1}, d_{2}, d_{3}\right)$.

Theorem 3.10. Let $P=F \cdot 1 \oplus N$ where $N$ is an associative commtative nil. algebra of type $\left(m, n, d_{1}, d_{2}, d_{3}\right)$ with $m>4$ over a field $F$ of char. $\neq 2,3$. If $P$ is nearly simple then $a^{2} b_{i} b_{j}=a b_{i} b_{j} b_{k}=b_{i} b_{j} b_{k} b_{l}=0$ for $i, j, k, l=1, \ldots, d_{1}$ -1 where $a, b_{i}$ 's are as in Theorem 3.8 and char $F$ divides $m$.

Proof. Suppose $P$ is nearly simple. Then there is an antiflexible map $\phi$ with $P(\phi)$ simple. Since $a^{m-1}$ is in $M$ and, for each $i, \phi\left(a^{m-1}, b_{i}\right)=0$ (Lemma 3.3), $\phi\left(a^{m-1}, a\right) \neq 0$. Considering $a, a, a, b_{i}, b_{j}$ and using Theorem 2.3 we get $\phi\left(a^{2} b_{i} b_{j}, a\right)=0$. But $\phi\left(a^{2} b_{i} b_{j}, a\right)=a_{i, j} \phi\left(a^{m-1}, a\right)$, so $\alpha_{i, j}=0$ for $i, j=1, \ldots$, $d_{1}-1$. Similarly it can be shown that $a b_{i} b_{j} b_{k}=0$ and $b_{i} b_{j} b_{k} b_{l}=0$ for $i, j, k$, $l=1, \cdots, d_{1}-1$. By Theorem 2.3, $m \phi\left(a^{m-1}, a\right)=0$. Therefore, char $F$ divides $m$.

4. Algebras with $N \cdot N \cdot N=0$. We have determined all nodal simple antiflexible algebras of type $(n-2, n)$ with $n-2>3$. In this section we get a few preliminary results for algebras of class 3 and then determine all nodal simple totally antiflexible algebras of types $(3, ;)$ and $(3,6)$. These are the only types discussed in [10].

Let $N$ denote a commutative associative nilalgebra of class 3 over a field $F$ and let $n-1$ denote the dimension of $N$. If $v_{1}, \cdots, v_{q}$ is a basis of $M$ (the annihilator of $N)$ we write a basis for the algebra $N$ in the form $\left\{u_{1}, \cdots, u_{r}\right.$, 
$\left.v_{1}, \cdots, v_{q}\right\}$. Since $N$ is of class $3, N^{2} \subseteq M$. Therefore $u_{i} u_{j}=\sum_{k=1}^{q} a_{i, j}^{k} v_{k}$ where $a_{i, j}^{k^{q}}=a_{j, i}^{k}$ is in $F$.

Up to isomorphism, the algebra $N$ is given by $q r^{2}$ elements $a_{i, j}^{k}$ or $q$ symmetric matrices $A^{(k)}=\left(\alpha_{i, j}^{k}\right)$ of degree $r$. We shall call the matrices $A^{(k)}$ the structural matrices of the algebra $N$. It is known [10] that if we change the basis of $N$, the new structural matrices are congruent to the one obtained previously.

Since $N^{2} \subseteq M \subseteq N$, there is a basis $\left\{u_{1}, \cdots, u_{s}, v_{1}, \ldots, v_{l}, w_{1}, \ldots, w_{t}\right\}$ of $N$ such that $\left\{v_{1}, \ldots, v_{l}\right\}$ is a basis of $N^{2}$ and $\left\{v_{1}, \ldots, v_{l}, w_{1}, \ldots, w_{t}\right\}$ is a basis of $M$. Let $V$ be the space spanned by $\left\{u_{1}, \ldots, u_{s}, v_{1}, \ldots, v_{l}\right\}$ and $W$ be the space spanned by $\left\{w_{1}, \cdots, w_{t}\right\}$. Then $N=V+W, V \cdot W=W^{2}=0$ and the annihilator of $V$ is $V^{2}$. Hence $N=V \oplus W$ where $V$ is of class 3 and $W$ is of class 2. Since commutative nilalgebras $N$ of class 2 have been determined [10], the description of commutative nilalgebras of class 3 reduces to the case in which $M=N^{2}$.

Lemma 4.1. Let $A=V \oplus W$ be an associative commutative algebra over a field of char. $\neq 2,3$. If $\phi_{1}$ and $\phi_{2}$ are antiflexible maps on $V$ and $W$ respectively then there exists an antiflexible map $\phi$ on $A$ such that $\phi\left(v, v^{\prime}\right)=\phi_{1}\left(v, v^{\prime}\right)$ for all $v, v^{\prime}$ in $V$ and $\phi\left(w, w^{\prime}\right)=\phi_{2}\left(w, w^{\prime}\right)$ for all $w, w^{\prime}$ in $W$.

Proof. Let $x, y$ be in $A$; then there exists $v_{1}, v_{2}$ in $V$ and $w_{1}, w_{2}$ in $W$ such that $x=v_{1}+w_{1}$ and $y=v_{2}+w_{2}$. Define $\phi$ on $A$ as follows:

$$
\begin{aligned}
& \phi(x, y)=\phi_{1}\left(v_{1}, v_{2}\right)+\phi_{2}\left(w_{1}, w_{2}\right), \\
& \phi(v, w)=\phi(w, v)=0 \text { for } v \text { in } V \text { and } w \text { in } W .
\end{aligned}
$$

Then it is easy to verify that $\phi$ is an antiflexible map.

In a similar way we prove the following lemma. We need first to define $\left.\phi\right|_{V}$. If $A=V+W$ and $\phi$ is a map on $A$, define $\left.\phi\right|_{V}$ by $\left.\phi\right|_{V}\left(v_{1}+w_{1}, v_{2}+w_{2}\right)=$ $\phi\left(v_{1}, v_{2}\right)$ for $v_{1}, v_{2}$ in $V$ and $w_{1}, w_{2}$ in $W$.

Lemma 4.2. Let $A=V \oplus W$ be an associative commutative algebra over a field $F$. If $\phi$ is an antiflexible map on $A$ sucb that $\phi(v, w)=0, \phi\left(v_{1}, v_{2}\right)$ is in $V$ and $\phi\left(w_{1}, w_{2}\right)$ is in $W$ for $v, v_{1}, v_{2}$ in $V$ and $w, w_{1}, w_{2}$ in $W$ then $\phi=\left.\phi\right|_{V}+\left.\phi\right|_{W} ;\left.\phi\right|_{V}$, $\left.\phi\right|_{W}$ are antiflexible maps on $V$ and $W$ respectively.

Proof. We need to show that $\phi=\left.\phi\right|_{V}+\left.\phi\right|_{W}$. Let $x, y$ be in $A$. Then $x=$ $v_{1}+w_{1}, y=v_{2}+w_{2}$ for some $v_{1}, v_{2}$ in $V$ and for some $w_{1}, w_{2}$ in $W$. Since $\phi$ is bilinear on $A$,

$$
\begin{aligned}
\phi(x, y) & =\phi\left(v_{1}, v_{2}\right)+\phi\left(v_{1}, w_{2}\right)+\phi\left(w_{1}, v_{1}\right)+\phi\left(w_{1}, w_{2}\right) \\
& =\left.\phi\right|_{V}(x, y)+\left.\phi\right|_{W}(x, y) .
\end{aligned}
$$

Now suppose $M=N^{2}$. Then the matrices $A^{(1)}, \ldots, A^{(q)}$ are linearly independent and it follows that two commutative nilpotent algebras of class 3 over a 
field $F$ are isomorphic if and only if corresponding spaces of bilinear forms determined by the matrices of the algebra are equivalent [10]. The proof of the following two lemmas are also found in [10].

Lemma 4.3. If $(1 / 2) r(r+1)=q$ then up to isomorphism there is exactly one associative commutative nilalgebra $N$ of class 3 over a field $F$ such that the $\operatorname{dim} N$ over $F$ is $q+r$ and $M=N^{2}$.

Lemma 4.4. If $M=N^{2}$ then $q \leq(1 / 2) r(r+1)$.

Lemma 4.5. Let $P=F \cdot 1 \oplus N$ where $N$ is an associative commutative nilalge. bra of type $(3, n)$ over a splitting field $F$ of char. $\neq 2,3$ with $M=N^{2}$. If $v_{1}, \cdots$, $v_{q}, u_{1}, \therefore, u_{r}$ is a basis of $N$ sucb that $v_{1}, \ldots, v_{q}$ is a bas is of $M$ and, for each $i, u_{\mathrm{i}}^{2}=\Sigma_{j \neq i} a_{i, j} v_{j}$, then $P$ is nearly simple.

Proof. Define $\phi$ on the basis of $P$ as follows:

$$
\begin{gathered}
\phi\left(v_{i}, v_{j}\right)=\delta_{i, j}=-\phi\left(u_{j}, v_{i}\right) \text { where } \delta_{i j}= \begin{cases}0 & \text { if } i \neq j, \\
1 & \text { if } i=j .\end{cases} \\
\phi(x, y)=0 \text { for any other pair of basis elements } x \text { and } y .
\end{gathered}
$$

Extend $\phi$ bilinearly to all of $P \times P$. Then it is routine to verify that $\phi$ is an antiflexible map and $P(\phi)$ is simple.

Now we consider algebras of type $(3,5)$. Note that if $P$ is a nodal simple totally antiflexible algebra of type $(3,5)$ then it is either of type $(3,5,3)$ or of type $(3,5,2,2)$. By Theorem 2.10 , there is no nodal simple totally antiflexible algebra of type $(3,5,3)$ over a field of char. $\neq 2,3$.

Theorem 4.1. Let $P=F \cdot 1 \oplus N$ be an associative commutative nearly nodal algebra of type $(3,5,2,2)$ over a field $F$ of char. $\neq 2,3$. Then $P$ is nearly simple if and only if

(48) $N$ is spanned by $\left\{a, a^{2}, b, c\right\}$ with $b$ in $N_{1}^{\prime}, c$ in $N_{2}^{\prime}$ and $c$ is either $a b$ or $b^{2}$,

(49) if $c=a b$, then $b^{2}=\gamma a^{2}+\delta a b$ with $4 y+\delta^{2}=0$, and if $c=b^{2}$ then $a b$ $=\alpha a^{2}+\beta b^{2}$ with $4 \alpha \beta=1$.

Proof. Suppose $P$ is nearly simple. Then there is a $\phi$ with $P(\phi)$ simple. By Corollary 3.2, $N$ is spanned by $a, a^{2}, b, c$ with $b$ in $N_{1}^{\prime}, c$ in $N_{2}^{\prime}$ and $c$ is either $a b$ or $b^{2}$. Note that $M$ is spanned by $a^{2}, a b, b^{2}$. So $\phi\left(a^{2}, b\right) \neq 0 \neq \phi\left(b^{2}, a\right)$ and, by Lemma 3.3, $\phi(a b, a)=(-1 / 2) \phi\left(a^{2}, b\right) \neq 0, \phi(a b, b)=(-1 / 2) \phi\left(b^{2}, a\right) \neq 0$. If $c=a b$ then since $b^{2}$ is in $N_{2}, b^{2}=\gamma a^{2}+\delta a b$, for some $\gamma, \delta$ in $F$. Therefore, $\phi\left(b^{2}, a\right)=\delta \phi(a b, a)$ or equivalently $-2 \phi(a b, b)=(-1 / 2) \delta \phi\left(a^{2}, b\right)$. Now

$$
0=4 \phi\left(b^{2}, b\right)=4 \gamma \phi\left(a^{2}, b\right)+4 \delta \phi(a b, b)=\left(4 \gamma+\delta^{2}\right) \phi\left(a^{2}, b\right) .
$$

Since $\phi\left(a^{2}, b\right) \neq 0$, we have $4 \gamma+\delta^{2}=0$. 
On the other hand if $c=b^{2}$ then $a b=\alpha a^{2}+\beta b^{2} ; \alpha, \beta$ are in $F$. So $\phi(a b, a)=\beta \phi\left(b^{2}, a\right)$ or equivalently $(-1 / 2) \phi\left(a^{2}, b\right)=-2 \beta \phi(a b, b)$. Thus $\phi(a b, b)=a \phi\left(a^{2}, b\right)=4 \alpha \beta \phi(a b, b)$ which implies that $4 \alpha \beta=1$.

Conversely, suppose $P$ satisfies (48) and (49). If $c=a b$, define $\phi$ on the basis of $P$ as follows:

$$
\begin{aligned}
& \phi\left(a^{2}, b\right)=4=-\phi\left(b, a^{2}\right), \quad \phi(a b, a)=-2=-\phi(a, a b), \\
& \phi(a b, b)=\delta=-\phi(b, a b), \quad \phi\left(b^{2}, a\right)=-2 \delta=-\phi\left(a, b^{2}\right), \\
& \phi(x, y)=0 \quad \text { for any other pair of basis elements } x \text { and } y .
\end{aligned}
$$

Extend $\phi$ bilinearly to $P \times P$. Then it is easy to verify that $\phi$ is antiflexible and $P(\phi)$ is simple.

If $c=b^{2}$, we define $\phi$ on the basis of $P$ as follows:

$$
\begin{array}{ll}
\phi\left(a^{2}, b\right)=2=-\phi\left(b, a^{2}\right), & \phi(a b, a)=-1=-\phi(a, a b), \\
\phi(a b, b)=2 a=-\phi(b, a b), & \phi\left(b^{2}, a\right)=-4 a=-\phi\left(a, b^{2}\right), \\
\phi(x, y)=0 & \text { otherwise. }
\end{array}
$$

Extend $\phi$ bilinearly to all of $P \times P$. Then $\phi$ is antiflexible and $P(\phi)$ is simple.

Theorem 4.2. Let $P=F \cdot 1 \oplus N$ be an associative, commutative, nearly nodal, nearly simple algebra of type $(3,5,2,2)$ over a field $F$ of char. $\neq 2,3$. If $\phi$ is an antiflexible map on $P$, then $P(\phi)$ is simple if and only if $H(\phi) \subseteq F \cdot 1$ and $\phi\left(a^{2}, b\right) \neq 0$.

Proof. By Theorem 4.1,N is spanned by $a, a^{2}, b, c$ where $c$ is either $a b$ or $b^{2}$. Also, if $c=a b$ then $b^{2}=\gamma a^{2}+\delta a b$ with $4 y+\delta^{2}=0$, and if $c=b^{2}$ then $a b=\alpha a^{2}+\beta b^{2}$ with $4 \alpha \beta=1$.

Now assume $P(\phi)$ is simple. Then since $a^{2}$ and $b^{2}$ are each in $M$ we have $\phi\left(a^{2}, b\right) \neq 0$ and $\phi\left(b^{2}, a\right) \neq 0$. By Lemma $3.2, \phi(a b, a) \neq 0 \neq \phi(a b, b)$. Let $x, y$ be in $P$; then $\phi(x, y)=a_{0}+a_{1} a+a_{2} a^{2}+a_{3} b+a_{4} c$. Since $\phi$ is an antiflexible map, we have $0=\phi\left(\phi(x, y), a^{2}\right)=a_{3} \phi\left(b, a^{2}\right)$. Thus $\alpha_{3}=0$. Also, $0=\phi\left(\phi(x, y), b^{2}\right)$ $=a_{1} \phi\left(a, b^{2}\right)$ which implies $\alpha_{1}=0$. Therefore, $\phi(x, y)=a_{0}+Y$ where $Y=$ $a_{2} a^{2}+a_{4} c$ is in $M$ and, for any $z$ in $P, 0=\phi(\phi(x, y), z)=\phi(Y, z)$. Hence $Y=0$ and so $\phi(x, y)=\alpha_{0}$ is in $F \cdot 1$.

Conversely, suppose $H(\phi) \subseteq F \cdot 1$ and $\phi\left(a^{2}, b\right)=4 \eta \neq 0$. Note that $M$ is spanned by $a^{2}, c$. If $c=a b$ then since $\phi$ is antiflexible, $\phi(a b, a)=-2 \eta$, $\phi\left(b^{2}, a\right)=-2 \delta \eta$ and $\phi(a b, b)=\delta \eta$. Let $x$ be a nonzero element of $M$; then $x=$ $\mu a^{2}+\nu a b$. If $\nu \neq 0$, then $\phi(x, a) \neq 0$, and if $\nu=0$ then $\phi(x, b) \neq 0$. Hence (17) is satisfied. Similarly if $c=b^{2}$, it can be shown that for each nonzero $x$ in $M$ there is a $y$ in $N$ with $\phi(x, y) \neq 0$. Hence in either case, by Theorem 2.5, $P(\phi)$ is simple. 
In the rest of this section we will restrict ourselves to the nodal algebras of type $(3,6)$ over the field of complex numbers. The proof of the following theorem is found in [10].

Theorem 4.3. The set of all associative commutative nilalgebras of type $(3,6)$ over the field of complex numbers contains only 13 algebras that are distinct up to isomorphism:

$$
\begin{aligned}
& \left.P_{1}=\left[u_{1}, u_{2}, u_{3}, u_{1}^{2}, u_{2}^{2}\right] \text { (algebra spanned by } u_{1}, u_{2}, u_{3}, u_{1}^{2} \text { and } u_{2}^{2}\right), u_{3}^{2}= \\
& u_{1}^{2}+u_{2}^{2}, u_{j} u_{k}=0 \text { for } j, k=1,2,3 ; \\
& P_{2}=\left[u_{1}, u_{2}, u_{3}, u_{1}^{2}, u_{3}^{2}\right], u_{2}^{2}=2 u_{3}^{2}-u_{1}^{2}, u_{j} u_{k}=0 \text { for } j \neq k ; j, k=1,2,3 ; \\
& P_{3}=\left[u_{1}, u_{2}, u_{3}, u_{1}^{2}, u_{2} u_{3}\right], u_{2}^{2}=u_{1}^{2}+i u_{2} u_{3}, u_{3}^{2}=u_{1}^{2}-i u_{2} u_{3}, u_{1} u_{2}=u_{1} u_{3}=0 ; \\
& P_{4}=\left[u_{1}, u_{2}, u_{3}, u_{1}^{2}, u_{2} u_{3}\right], u_{2}^{2}=u_{1}^{2}+(i-1) u_{2} u_{3}, u_{3}^{2}=u_{1}^{2}-(i+1) u_{2} u_{3}, u_{1} u_{2} \\
& =u_{1} u_{3}=0 ; \\
& P_{5}=\left[u_{1}, u_{2}, u_{3}, u_{1}^{2}, u_{1} u_{2}\right], u_{2}^{2}=u_{1}^{2}=u_{3}^{2}, u_{2} u_{3}=-i u_{1} u_{2}, u_{1} u_{3}=0 ; \\
& P_{6}=\left[u_{1}, u_{2}, u_{3}, u_{1}^{2}, u_{1} u_{3}\right], u_{2}^{2}=u_{1}^{2}, u_{2} u_{3}=i u_{1} u_{3}, u_{1} u_{2}=u_{3}^{2}=0 ; \\
& P_{7}=\left[u_{1}, u_{2}, u_{3}, u_{4}, u_{1}^{2}\right], u_{2}^{2}=u_{3}^{2}=u_{4}^{2}=u_{1}^{2}, u_{j} u_{k}=0 \text { for } j \neq k ; j, k=1,2,
\end{aligned}
$$

$P_{8}=\left[u_{1}, u_{2}, u_{1}^{2}, u_{1} u_{2}, u_{2}^{2}\right]$

$P_{9}=\left[u, u^{2}, v_{1}, v_{2}, v_{3}\right], v_{j}$ in $M, u v_{j}=v_{j} v_{k}=0$ for $j, k=1,2,3$;

$P_{10}=\left[u_{1}, u_{2}, u_{1}^{2}, v_{1}, v_{2}\right], v_{j}$ is in $M, u_{2}^{2}=u_{1}^{2}, u_{j} v_{k}=0$ for $j, k=1,2, u_{1} u_{2}=0$;

$P_{11}=\left[u_{1}, u_{2}, u_{3}, u_{1}^{2}, v\right], u_{1}^{2}=u_{2}^{2}=u_{3}^{2}, u_{j} u_{k}=u_{j} v=0$ for $j \neq k ; j, k=1,2,3$; $v$ in $M$;

$P_{12}=\left[u_{1}, u_{2}, u_{1}^{2}, u_{2}^{2}, v\right], v$ in $M, u_{1} u_{2}=u_{1} v=u_{2} v=0$
$P_{13}=\left[u_{1}, u_{2}, u_{1}^{2}, u_{1} u_{2}, v\right], u_{2}^{2}=u_{1}^{2}-2 i u_{1} u_{2}, u_{1} v=u_{2} v=0$.

Using this we have the following result.

Theorem 4.4. Let $P=C \cdot 1 \oplus N$ where $N$ is an associative, commutative, nilalgebra of type $(3,6)$ over the field of complex numbers $C$. Then $P$ is nearly simple if and only if $N=P_{6}$.

Proof. Suppose $P$ is nearly simple, then there is an antiflexible map $\phi$ with $P(\phi)$ simple.

If $N=P_{1}=\left[u_{1}, u_{2}, u_{3}, u_{1}^{2}, u_{2}^{2}\right], u_{3}^{2}=u_{1}^{2}+u_{2}^{2}, u_{1} u_{2}=u_{2} u_{3}=u_{3} u_{1}=0$, then $\phi\left(u_{1} u_{2}, u_{1}\right)=\phi\left(u_{1} u_{3}, u_{1}\right)=0$. So by Lemma 3.2, $\phi\left(u_{1}^{2}, u_{2}\right)=\phi\left(u_{1}^{2}, u_{3}\right)=0$. Hence $\phi\left(u_{1}^{2}, z\right)=0$ for all $z$ in $P$. This is impossible as $u_{1}^{2}$ is a nonzero element of $M$ and so $N \neq P_{1}$. The same reasoning also proves that $N$ cannot be any one of $P_{2}, P_{3}$ and $P_{4}$.

So assume that $N=P_{5}=\left[u_{1}, u_{2}, u_{3}, u_{1}^{2}, u_{1} u_{2}\right], u_{1}^{2}=u_{2}^{2}=u_{3}^{2}, u_{2} u_{3}=i u_{1} u_{2}$, $u_{1} u_{3}=0$. Then $\phi\left(u_{1}^{2}, u_{2}\right)=\phi\left(u_{2}^{2}, u_{2}\right)=0$ and $\phi\left(u_{1}^{2}, u_{3}\right)=\phi\left(u_{3}^{2}, u_{3}\right)=0$. Consequently, $\phi\left(u_{1}^{2}, z\right)=0$ for all $z$ in $P$. Since $u_{1}^{2}$ is in $M, u_{1}^{2}=0$. This is impossible, so $N \neq P_{5}$.

Now suppose $N=P_{7}=\left[u_{1}, u_{2}, u_{3}, u_{4}, u_{1}^{2}\right], u_{1}^{2}=u_{2}^{2}=u_{3}^{2}=u_{4}^{2}, u_{j} u_{k}=0$ for 
$j \neq k ; j, k=1,2,3,4$. Then $P$ is of type $(3,6,4)$. But by Theorem 2.10 there is no nearly simple algebra of type $(3,6,4)$. Thus $N \neq P_{7}$.

If $N=P_{8}=\left[u_{1}, u_{2}, u_{1}^{2}, u_{1} u_{2}, u_{2}^{2}\right]$, then since $u_{1}^{2}, u_{2}^{2}$ are in $M, \phi\left(u_{1}^{2}, u_{2}\right) \neq 0$ and $\phi\left(u_{2}^{2}, u_{1}\right) \neq 0$. Also, by Lemma $3.2, \phi\left(u_{1}^{2}, u_{2}\right)+2 \phi\left(u_{1} u_{2}, u_{1}\right)=0$ and $\phi\left(u_{2}^{2}, u_{1}\right)+2 \phi\left(u_{1} u_{2}, u_{2}\right)=0$. Therefore, $\phi\left(u_{1} u_{2}, u_{1}\right) \neq 0 \neq \phi\left(u_{1} u_{2}, u_{2}\right)$. We will first show that $H(\phi) \subseteq C \cdot 1$. Let $x, y$ be in $P$, then

$$
\phi(x, y)=\alpha_{0}+\alpha_{1} u_{1}+\alpha_{2} u_{2}+\alpha_{3} u_{1}^{2}+\alpha_{4} u_{1} u_{2}+\alpha_{5} u_{2}^{2} \text {, }
$$

$\alpha_{i}$ in $C$ for $i=0,1,2,3,4,5$. Since $\phi$ is an antiflexible map, $0=\phi\left(\phi(x, y), u_{2}^{2}\right)$ $=\alpha_{1} \phi\left(u_{1}, u_{2}^{2}\right)$ which implies $\alpha_{1}=0$. Also, $0=\phi\left(\phi(x, y), u_{1}^{2}\right)=a_{2} \phi\left(u_{2}, u_{1}^{2}\right)$ implies that $\alpha_{2}=0$. Therefore, $\phi(x, y)=\alpha_{0}+Y$ where $Y=\alpha_{3} u_{1}^{2}+\alpha_{4} u_{1} u_{2}+$ $\alpha_{5} u_{2}^{2}$ is in $M$. Now for any $z$ in $P, 0=\phi(\phi(x, y), z)=\phi(Y, z)$ so $Y=0$. Hence $H(\phi) \subseteq C \cdot 1$. Suppose $\phi\left(u_{1} u_{2}, u_{1}\right)=\alpha \neq 0$ and $\phi\left(u_{1} u_{2}, u_{2}\right)=\beta \neq 0$. Then by Lemma 3.2, $\phi\left(u_{1}^{2}, u_{2}\right)=-2 \alpha$ and $\phi\left(u_{2}^{2}, u_{1}\right)=-2 \beta$. Let $x=2 \beta u_{1} u_{2}+\alpha u_{2}^{2}+$ $\beta^{2} / \alpha u_{1}^{2}$. Then $x$ is a nonzero element of $M$ and $\phi\left(x, u_{1}\right)=\phi\left(x, u_{2}\right)=0$. Hence for all $z$ in $P, \phi(x, z)=0$ and so $x=0$. This is a contradiction and so $N \neq P_{8}$.

Let $N=P_{9}=\left[u, u^{2}, v_{1}, v_{2}, v_{3}\right], v_{j}$ in $M, u v_{j}=v_{j} v_{k}=0$ for $j, k=1,2,3$. Since $u^{2}$ is in $N_{2}, \phi\left(u^{2}, v_{j}\right)=0$ for $j=1,2,3$. Hence $u^{2}=0$ which is impossible. Thus $N \neq P_{9}$.

Next suppose $N=P_{10}=\left[u_{1}, u_{2}, u_{1}^{2}, v_{1}, v_{2}\right], v_{j}$ in $M, u_{1}^{2}=u_{2}^{2}, u_{1} u_{2}=0=$ $u_{j} v_{k}$ for $j, k=1,2$. Then $\phi\left(u_{1}^{2}, u_{j}\right)=\phi\left(u_{1}^{2}, v_{j}\right)=0$ for $j=1,2$. Consequently, $u_{1}^{2}=0$ which is a contradiction.

Now assume $N=P_{11}=\left[u_{1}, u_{2}, u_{3}, u_{1}^{2}, v\right], v$ is in $M, u_{1}^{2}=u_{2}^{2}=u_{3}^{2}, u_{j} u_{k}=$ $u_{j} v=0$ for $j \neq k ; j, k=1,2,3$. By Theorem 2.1 it follows that $\phi\left(u_{1}^{2}, u_{1}\right)=$ $\phi^{j}\left(u_{1}^{2}, v_{j}\right)=0$ for $j=1,2,3$. Since $u_{1}^{2}$ is in $M, u_{1}^{2}=0$. This is impossible, so $N \neq P_{11}$.

If $N=P_{12}=\left[u_{1}, u_{2}, u_{1}^{2}, u_{2}^{2}, v\right], v$ is in $M, u_{1} u_{2}=u_{j} v=0$ for $j=1,2$, then $\phi\left(u_{1}^{2}, u_{j}\right)=-2 \phi\left(u_{1} u_{j}, u_{1}\right)=0, \phi\left(u_{1}^{2}, v\right)=-2 \phi\left(u_{1} v, u_{1}\right)=0$ for $j=1,2$. Therefore $u_{1}^{2}=0$, a contradiction.

Finally, suppose $N=P_{13}=\left[u_{1}, u_{2}, u_{1}^{2}, u_{1} u_{2}, v\right], v$ in $M, u_{2}^{2}=u_{1}^{2}-2 i u_{1} u_{2}$, $u_{j} v=0$ for $j=1,2$. Then $H(\phi) \subseteq C$. For, if $x, y$ are in $P$, then $\phi(x, y)=$ $a_{0}+a_{1} u_{1}+a_{2} u_{2}+a_{3} u_{1}^{2}+\alpha_{4} u_{1} u_{2}+\alpha_{5} v, a_{j}$ in $C, j=0,1,2,3,4,5$. Since $P(\phi)$ is simple, $\phi\left(u_{1}^{2}, u_{2}\right) \neq 0, \phi\left(u_{1} u_{2}, u_{1}\right) \neq 0 \neq \phi\left(u_{1} u_{2}, u_{2}\right)$, and either $\phi\left(v, u_{1}\right)$ $\neq 0$ or $\phi\left(v, u_{2}\right) \neq 0$. Now $0=\phi\left(\phi(x, y), u_{1}^{2}\right)=\alpha_{2} \phi\left(u_{2}, u_{1}^{2}\right)$ which implies $\alpha_{2}=0$. Similarly, $0=\phi\left(\phi(x, y), u_{1} u_{2}\right)=a_{1} \phi\left(u_{1}, u_{1} u_{2}\right)$ implies $a_{1}=0$. Thus we have $\phi(x, y)=a_{0}+Y$ where $Y=a_{3} u_{1}^{2}+a_{4} u_{1} u_{2}+a_{5} v$ is in $M$. For any $z$ in $P, 0=$ $\phi(\phi(x, y), z)=\phi(Y, z)$ so $Y=0$. Hence $\phi(x, y)$ is in $C \cdot 1$. Let $\phi\left(u_{1} u_{2}, u_{1}\right)=$ $\alpha \neq 0, \phi\left(v, u_{1}\right)=\beta, \phi\left(v, u_{2}\right)=\gamma$. Then $\phi\left(u_{1}^{2}, u_{2}\right)=-2 \alpha, \phi\left(u_{2}^{2}, u_{1}\right)=-2 i \alpha$ and $\phi\left(u_{1} u_{2}, u_{2}\right)=-i \alpha$. If $\beta=0$, define $x=\gamma u_{1}^{2}+2 \alpha v, x \neq 0, x$ is in $M$. Then 
$\phi\left(x, u_{1}\right)=0=\phi\left(x, u_{2}\right)$. Consequently, $\phi(x, z)=0$ for all $z$ in $P$ and so $x=0$, which is impossible. On the other hand, if $\beta \neq 0$, define $x=2 \beta u_{1} u_{2}-2 \alpha v+$ $(i \beta-\gamma) u_{1}^{2}$. Then $x$ is a nonzero element in $M$ and $\phi\left(x, u_{1}\right)=\phi\left(x, u_{2}\right)=0$. Hence $\phi(x, z)=0$ for all $z$ in $P$ and so $x=0$. This is a contradiction.

Thus the only possibility left up to isomorphism is $N=P_{6}=\left[u_{1}, u_{2}, u_{3}, u_{1}^{2}\right.$, $\left.u_{1} u_{3}\right], u_{1}^{2}=u_{2}^{2}, u_{2} u_{3}=i u_{1} u_{3}, u_{3}^{2}=u_{1} u_{2}=0$. In this case $\phi\left(u_{1}^{2}, u_{2}\right)=\phi\left(u_{2}^{2}, u_{2}\right)=$ $0, \phi\left(u_{2}^{2}, u_{1}\right)=0$. Since $P(\phi)$ is simple, $\phi\left(u_{1}^{2}, u_{3}\right)=\phi\left(u_{2}^{2}, u_{3}\right)=-2 \phi\left(u_{1} u_{3}, u_{1}\right) \neq$ 0 . By Lemma 3.2, $0=\phi\left(u_{2}^{2}, u_{3}\right)+2 \phi\left(u_{2} u_{3}, u_{2}\right)=\phi\left(u_{1}^{2}, u_{3}\right)+2 i \phi\left(u_{1} u_{3}, u_{2}\right)$ and, by Theorem 2.3, $0=\phi\left(u_{1} u_{2}, u_{3}\right)+\phi\left(u_{1} u_{3}, u_{2}\right)+\phi\left(u_{2} u_{3}, u_{1}\right)=\phi\left(u_{1} u_{3}, u_{2}\right)+$ $i \phi\left(u_{1} u_{3}, u_{1}\right)=\phi\left(u_{1} u_{3}, u_{2}+i u_{1}\right)$.

Conversely, suppose $P=C \cdot 1+P_{6}$. Define $\phi$ on the basis of $P$ as follows:

$$
\begin{gathered}
\phi\left(u_{1} u_{3}, u_{1}\right)=1=-\phi\left(u_{1}, u_{1} u_{3}\right), \\
\phi\left(u_{1}^{2}, u_{3}\right)=-2=-\phi\left(u_{3}, u_{1}^{2}\right), \\
\phi\left(u_{1} u_{3}, u_{2}\right)=-i=-\phi\left(u_{2}, u_{1} u_{3}\right),
\end{gathered}
$$

$\phi(x, y)=0$ for any other pair of basis elements $x$ and $y$.

Extend $\phi$ belinearly to all of $P \times P$. Then it is a routine to verify that $\phi$ is an antiflexible map and $P(\phi)$ satisfies (17). Hence, by Theorem 2.5,P( $\phi)$ is simple and the proof is complete.

Having characterized all nearly simple nodal algebras of type $(3,6)$ over the field of complex numbers we are now interested in finding all possible candidates for $\phi$.

Theorem 4.5. Let $P=C \cdot 1 \oplus P_{6}$ where $C$ is the basefield of complex num. bers and $P_{6}=\left[u_{1}, u_{2}, u_{3}, u_{1}^{2}, u_{1} u_{3}\right], u_{1}^{2}=u_{2}^{2}, u_{2} u_{3}=i u_{1} u_{3}, u_{3}^{2}=u_{1} u_{2}=0$ and let $\phi$ be an antiflexible map. Then $P(\phi)$ is simple if and only if

(50) for each $x, y$ in $P$ there exists $\alpha_{j}, j=0,1,2,4,5$, such that $\alpha_{1}=$ $i a_{2}$ and $\phi(x, y)=\alpha_{0}+\alpha_{1} u_{1}+\alpha_{2} u_{2}+\alpha_{4} u_{1}^{2}+\alpha_{5} u_{1} u_{3}$.

(51) $\phi\left(u_{1} u_{3}, u_{1}\right)=\beta_{0}+\beta_{1} u_{1}+\beta_{2} u_{2}+\beta_{4} u_{1}^{2}+\beta_{5} u_{1} u_{3}$ for some $\beta_{j}$ in $C, j=$ $0,1,2,4,5$, with $\beta_{1}=i \beta_{2}$ and $\beta_{0} \neq 0$.

Proof. Assume $P(\phi)$ is simple. Then $\phi\left(u_{1}^{2}, u_{3}\right)=-2 \phi\left(u_{1} u_{3}, u_{1}\right) \neq 0$. Let $x, y$ be in $P$, then $\phi(x, y)=a_{0}+a_{1} u_{1}+a_{2} u_{2}+a_{3} u_{3}+a_{4} u_{1}^{2}+a_{5} u_{1} u_{3}, a_{j}$ in $C$ for $j=0,1,2,3,4,5$. Since $\phi$ is an antiflexible map, $0=\phi\left(\phi(x, y), u_{1}^{2}\right)=$ $\alpha_{3} \phi\left(u_{3}, u_{1}^{2}\right)$. Therefore, $\alpha_{3}=0$. Also, $0=\phi\left(\phi(x, y), u_{1} u_{3}\right)=\alpha_{1} \phi\left(u_{1}, u_{1} u_{3}\right)+$ $\alpha_{2} \phi\left(u_{2}, u_{1} u_{3}\right)$ and $0=\phi\left(u_{1} u_{2}, u_{3}\right)+\phi\left(u_{1} u_{3}, u_{2}\right)+\phi\left(u_{2} u_{3}, u_{1}\right)=\phi\left(u_{1} u_{3}, u_{2}\right)+$ $i \phi\left(u_{1} u_{3}, u_{1}\right)$. Consequently, $\left(\alpha_{1}-i \alpha_{2}\right) \phi\left(u_{1} u_{3}, u_{1}\right)=0$ which implies $\alpha_{1}=i \alpha_{2}$. If $x=u_{1} u_{3}$ and $y=u_{1}$ we get $\phi\left(u_{1} u_{3}, u_{1}\right)=\beta_{0}+i \beta_{2} u_{1}+\beta_{2} u_{2}+\beta_{4} u_{1}^{2}+\beta_{5} u_{1} u_{3}$ for some $\beta_{j}, j=0,2,4,5$ in $C$. If $\beta_{0}=0$ then $\phi\left(u_{1} u_{3}, u_{1}\right)$ generates a proper ideal of $P(\phi)$ so $\beta_{0} \neq 0$. We observe here that for each $x, y$ in $P$ there is an $\alpha$ in $C$ 
such that $\phi(x, y)=a \phi\left(u_{1} u_{3}, u_{1}\right)$, so if $\phi(x, y) \neq 0$ then $\alpha_{0} \neq 0$.

Conversely suppose $\phi$ satisfies (50) and (51). Let $J$ be an ideal of $P(\phi)$ with $x$ in $J, x \neq 0$. Then $x=\gamma_{0}+\gamma_{1} u_{1}+\gamma_{2} u_{2}+\gamma_{3} u_{3}+\gamma_{4} u_{1}^{2}+\gamma_{5} u_{1} u_{3}, \gamma_{j}$ in $C$. If $\gamma_{0} \neq 0$ then $\left(1 / \gamma_{0}\right) x=1-z$ is in $J$ for some $z$ with $z^{3}=0$, and if $\gamma_{0}=0$, $\gamma_{3} \neq 0$ then $\left(-1 / 2 \gamma_{3} \beta_{0}\right) \phi\left(x, u_{1}^{2}\right)=1-z$ is in $J$. Now suppose $\gamma_{0}=\gamma_{3}=0$ and $\gamma_{2} \neq 0$. Then $\left(-1 / 2 \gamma_{2} \beta_{0}\right) \phi\left(x * u_{2}, u_{3}\right)=\left(-1 / 2 \beta_{0}\right) \phi\left(u_{2}^{2}, u_{3}\right)=1-z$ is in $J$. Let $\gamma_{j}=0$ for $j=0,2,3$ and $\gamma_{1} \neq 0$. Then $\left(-1 / \beta_{0} \gamma_{1}\right) \phi\left(x, u_{1} u_{3}\right)=\left(-1 / \beta_{0}\right) \phi\left(u_{1}, u_{1} u_{3}\right)$ $=1-z$. If $\gamma_{j}=0$ for $j=0,1,2,3, \gamma_{4} \neq 0$, then since $\phi\left(u_{1} u_{3}, u_{3}\right)=$ $(-1 / 2) \phi\left(u_{3}^{2}, u_{1}\right)=0,\left(-1 / 2 \gamma_{4} \beta_{0}\right) \phi\left(x, u_{3}\right)=1-z$ is in J. Finally, if $\gamma_{j}=0$ for $j=0,1,2,3,4$ and $\gamma_{5} \neq 0$, then $\left(1 / \gamma_{5} \beta_{0}\right) \phi\left(x, u_{1}\right)=1-z$ is in $J$.

Hence in all cases $1=(1-z)\left(1+z+z^{2}\right)$ is in $J$ and so $J=P(\phi)$. Therefore, $P(\phi)$ is simple and we are done.

\section{BIBLIOGRAPHY}

1. C. T. Anderson and D. L. Outcalt, On simple anti-flexible rings, J. Algebra 10 (1968), 310-320. MR $37 \sharp 6337$.

2. D. T. Finkbeiner II, Introduction to matrices and linear transformation, 2 nd ed., Freeman, San Francisco, Calif., 1966. MR 33 \#1315.

3. F. Kosier, On a class of nonflexible algebras, Trans. Amer. Math. Soc. 102 (1962), 299-318. MR 24 \#A3187.

4. D. J. Rodabaugh, A generalization of the flexible law, Trans. Amer. Math. Soc. 114 (1965), 468-487. MR $32 \sharp 2452$.

5. - Some new results on simple algebras, Pacific J. Math. 17 (1966), 311317. MR $35 \# 2934$.

6. - Antiflexible algebras which are not power-associative, Proc. Amer. Math. Soc. 17 (1966), 237-239. MR $32 \sharp 4168$.

7. - On semisimple antiflexible algebras, Portugal. Math. 26 (1967), 261-271. MR $41 \# 1822$.

8. - On antiflexible algebras, Trans. Amer. Math. Soc. 169 (1972), 219-235.

9. R. D. Schafer, An introduction to nonassociative algebras, Pure and Appl. Math., vol. 22, Academic Press, New York, 1966. MR 35 \#1643.

10. D. A. Suprunenko and R. I. Tyškevič, Commutative matrices, Nauka i Tehnika Press, Minsk, 1966; English transl., Academic Press, New York, 1968. MR 34 \#1356.

DEPARTMENT OF MATHEMATICS, UNIVERSITY OF MISSOURI, COLUMBIA, MISSOURI 65201 DEPARTMENT OF MATHEMATICS, INDIAN INSTITUTE OF TECHNOLOGY, KANPUR, INDIA 